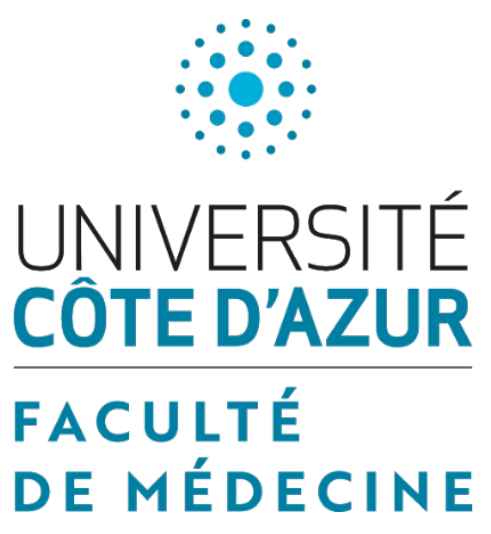

Thèse d'exercice de Médecine

Adrien Nicolau

Né le 22 juin 1992 à Tarbes

\title{
Anomalies biologiques hépatiques chez les patients sous nutrition parentérale d'introduction récente
}

Soutenue publiquement le 9 octobre 2020

A la Faculté de Médecine de Nice

\author{
Directeur de Thèse: Professeur Stéphane Schneider \\ Président du Jury: Professeur Thierry Piche \\ Assesseurs: Professeur Xavier Hébuterne \\ Professeur Geoffroy Vanbiervliet \\ Docteur Delphine Ouvrier
}




\title{
Liste des enseignants au 1er septembre 2020 à la Faculté de Médecine de Nice
}

\author{
Doyen \\ Pr. BAQUÉ Patrick
}

$\begin{array}{ll}\text { Vice-doyens } & \\ \text { Pédagogie } & \text { Pr. ALUNNI Véronique } \\ \text { Recherche } & \text { Pr DELLAMONICA jean } \\ \text { Etudiants } & \text { M. JOUAN Robin }\end{array}$

Chargé de mission projet Campus

Pr. PAQUIS Philippe

Conservateur de la bibliothèque Mme AMSELLE Danièle

Directrice administrative des services Mme CALLEA Isabelle

Doyens Honoraires

M. RAMPAL Patrick
M. BENCHIMOL Daniel

PROFESSEURS CLASSE EXCEPTIONNELLE

$\begin{array}{lll}\text { M. } & \text { BAQUÉ Patrick } & \text { Anatomie - Chirurgie Générale (42.01) } \\ \text { M. } & \text { BERNARDIN Gilles } & \text { Réanimation Médicale (48.02) } \\ \text { Mme } & \text { BLANC-PEDEUTOUR Florence } & \text { Cancérologie - Génétique (47.02) } \\ \text { M. } & \text { BOILEAU Pascal } & \text { Chirurgie Orthopédique et Traumatologique (50.02) } \\ \text { M. } & \text { DARCOURT Jacques } & \text { Biophysique et Médecine Nucléaire (43.01) } \\ \text { M. } & \text { DRICI Milou-Daniel } & \text { Pharmacologie Clinique (48.03) } \\ \text { M. } & \text { ESNAULT Vincent } & \text { Néphrologie (52-03) } \\ \text { M. } & \text { GILSON Éric } & \text { Biologie Cellulaire (44.03) } \\ \text { M. } & \text { GUGENHEIM Jean } & \text { Chirurgie Digestive (52.02) } \\ \text { M. } & \text { HASSEN KHODJA Reda } & \text { Chirurgie Vasculaire (51.04) } \\ \text { M. } & \text { HÉBUTERNE Xavier } & \text { Nutrition (44.04) } \\ \text { M. } & \text { HOFMAN Paul } & \text { Anatomie et Cytologie Pathologiques (42.03) } \\ \text { Mme } & \text { ICHAI Carole } & \text { Anesthésiologie et Réanimation Chirurgicale (48.01) } \\ \text { M. } & \text { LACOUR Jean-Philippe } & \text { Dermato-Vénéréologie (50.03) } \\ \text { M. } & \text { LEFTHERIOTIS Georges } & \text { Chirurgie vasculaire; médecine vasculaire (51.04) } \\ \text { M. } & \text { MARQUETTE Charles-Hugo } & \text { Pneumologie (51.01) } \\ \text { M. } & \text { MARTY Pierre } & \text { Parasitologie et Mycologie (45.02) } \\ \text { M. } & \text { MICHIELS Jean-François } & \text { Anatomie et Cytologie Pathologiques (42.03) } \\ \text { M. } & \text { MOUNIER Nicolas } & \text { Cancérologie, Radiothérapie (47.02) } \\ \text { M. } & \text { MOUROUX Jérôme } & \text { Chirurgie Thoracique et Cardiovasculaire (51.03) }\end{array}$




$\begin{array}{ll}\text { M. } & \text { PADOVANI Bernard } \\ \text { Mme } & \text { PAQUIS Véronique } \\ \text { M. } & \text { PAQUIS Philippe } \\ \text { M. } & \text { PRADIER Christian } \\ \text { M. } & \text { QUATREHOMME Gérald } \\ \text { M. } & \text { RAUCOULES-AIMÉ Marc } \\ \text { M. } & \text { ROBERT Philippe } \\ \text { M. } & \text { SCHNEIDER Stéphane } \\ \text { M. } & \text { TRAN Albert }\end{array}$

PROFESSEURS PREMIERE CLASSE

$\begin{array}{lll}\text { Mme } & \text { ASKENAZY-GITTARD Florence } & \text { Pédopsychiatrie (49.04) } \\ \text { M. } & \text { BARRANGER Emmanuel } & \text { Gynécologie Obstétrique (54.03) } \\ \text { M. } & \text { BÉRARD Étienne } & \text { Pédiatrie (54.01) } \\ \text { M. } & \text { BONGAIN André } & \text { Gynécologie-Obstétrique (54.03) } \\ \text { Mme } & \text { BREUIL Véronique } & \text { Rhumatologie (50.01) } \\ \text { M. } & \text { CASTILLO Laurent } & \text { O.R.L. (55.01) } \\ \text { M. } & \text { CHEVALLIER Patrick } & \text { Radiologie et Imagerie Médicale (43.02) } \\ \text { M. } & \text { DE PERETTI Fernand } & \text { Anatomie-Chirurgie Orthopédique (42.01) } \\ \text { M. } & \text { FERRARI Émile } & \text { Cardiologie (51.02) } \\ \text { M. } & \text { FERRERO Jean-Marc } & \text { Cancérologie ; Radiothérapie (47.02) } \\ \text { M. } & \text { FONTAINE Denys } & \text { Neurochirurgie (49.02) } \\ \text { M. } & \text { GUÉRIN Olivier } & \text { Méd. In; Gériatrie (53.01) } \\ \text { M. } & \text { HANNOUN-LEVI Jean-Michel } & \text { Cancérologie ; Radiothérapie (47.02) } \\ \text { M } & \text { JEAN BAPTISTE Elixène } & \text { Chirurgie vasculaire (51.04) } \\ \text { M. } & \text { LEVRAUT Jacques } & \text { Médecine d'urgence (48.05) } \\ \text { M. } & \text { LONJON Michel } & \text { Neurochirurgie (49.02) } \\ \text { M. } & \text { PASSERON Thierry } & \text { Dermato-Vénéréologie (50-03) } \\ \text { M. } & \text { PICHE Thierry } & \text { Gastro-entérologie (52.01) } \\ \text { Mme } & \text { RAYNAUD Dominique } & \text { Hématologie (47.01) } \\ \text { M. } & \text { ROUX Christian } & \text { rhumatologie (50.01) } \\ \text { M. } & \text { ROSENTHAL Éric } & \text { Médecine Interne (53.01) } \\ \text { M. } & \text { STACCINI Pascal } & \text { Biostatistiques et Informatique Médicale (46.04) } \\ \text { M. } & \text { THOMAS Pierre } & \text { Neurologie (49.01) } \\ \text { M. } & \text { TROJANI Christophe } & \text { Chirurgie Orthopédique et Traumatologique } \\ & & \text { (50.02) }\end{array}$

\section{PROFESSEURS DEUXIEME CLASSE}

$\begin{array}{lll}\text { Mme } & \text { ALUNNI Véronique } & \text { Médecine Légale et Droit de la Santé (46.03) } \\ \text { M. } & \text { ANTY Rodolphe } & \text { Gastro-entérologie (52.01) } \\ \text { M. } & \text { BAHADORAN Philippe } & \text { Cytologie et Histologie (42.02) } \\ \text { Mme } & \text { BAILLIF Stéphanie } & \text { Ophtalmologie (55.02) } \\ \text { Mme } & \text { BANNWARTH Sylvie } & \text { Génétique (47.04) } \\ \text { M. } & \text { BENIZRI Emmanuel } & \text { Chirurgie Générale (53.02) } \\ \text { M. } & \text { BENOIT Michel } & \text { Psychiatrie (49.03) } \\ \text { M. } & \text { BERTHET Jean-Philippe } & \text { Chirurgie Thoracique (51-03) } \\ \text { M. } & \text { BOZEC Alexandre } & \text { ORL- Cancérologie (47.02) } \\ \text { M. } & \text { BREAUD Jean } & \text { Chirurgie Infantile (54-02) } \\ \text { M. } & \text { BRONSARD Nicolas } & \text { Anatomie Chirurgie Orthopédique et Traumatologique } \\ & & \text { (42.01) } \\ \text { Mme } & \text { BUREL-VANDENBOS Fanny } & \text { Anatomie et Cytologie pathologiques (42.03) }\end{array}$




$\begin{array}{ll}\text { M. } & \text { CHEVALIER Nicolas } \\ \text { Mme } & \text { CHINETTI Giulia } \\ M . & \text { CLUZEAU Thomas } \\ M . & \text { DELLAMONICA Jean } \\ M . & \text { DELOTTE Jérôme } \\ \text { Mme } & \text { ESTRAN-POMARES Christelle } \\ M & \text { FAVRE Guillaume } \\ M . & \text { FOURNIER Jean-Paul } \\ \text { Mme } & \text { GIORDANENGO Valérie } \\ \text { Mme } & \text { GIOVANNINI-CHAMI Lisa } \\ M . & \text { IANNELLI Antonio } \\ M . & \text { ILIE Marius } \\ M . & \text { ORBAN Jean-Christophe } \\ M . & \text { ROHRLICH Pierre } \\ M . & \text { RUIMY Raymond } \\ M m e & \text { SACCONI Sabrina } \\ M m e & \text { SEITZ-POLSKI barbara } \\ M . & \text { VANBIERVLIET Geoffroy }\end{array}$

Endocrinologie, Diabète et Maladies Métaboliques (54.04)

Biochimie-Biologie Moléculaire (44.01)

Hématologie (47.01)

réanimation médicale (48.02)

Gynécologie-obstétrique (54.03)

Parasitologie et mycologie (45.02)

Néphrologie (44-02)

Thérapeutique (48-04)

Bactériologie-Virologie (45.01)

Pédiatrie (54.01)

Chirurgie Digestive (52.02)

Anatomie et Cytologie pathologiques (42.03)

Anesthésiologie-réanimation ; Médecine d'urgence (48.01)

Pédiatrie (54.01)

Bactériologie-virologie (45.01)

Neurologie (49.01)

Immunologie (47.03)

Gastro-entérologie (52.01)

\section{MAITRES DE CONFÉRENCES DES UNIVERSITÉS - PRATICIENS HOSPITALIERS}

M. AMBROSETTI Damien

Mme BERNARD-POMIER Ghislaine

M. CAMUZARD Olivier

Mme CONTENTI-LIPRANDI Julie

M. DOGLIO Alain

M. DOYEN Jérôme

M. FOSSE Thierry

M. GARRAFFO Rodolphe

Mme HINAULT Charlotte

M. HUMBERT Olivier

Mme LAMY Brigitte

Mme LONG-MIRA Elodie

$M$. LOTTE Romain

Mme MAGNIÉ Marie-Noëlle

M. MASSALOU Damien

Mme MOCERI Pamela

M. MONTAUDIE Henri

Mme MUSSO-LASSALLE Sandra

M. NAIIMI Mourad

M. SAVOLDELLI Charles

M. SQUARA Fabien

M. TESTA Jean

Mme THUMMLER Susanne

M. TOULON Pierre

M. TRAN Antoine
Cytologie et Histologie (42.02)

Immunologie (47.03)

Chirurgie Plastique (50-04)

Médecine d'urgence (48-04)

Bactériologie-Virologie (45.01)

Radiothérapie (47.02)

Bactériologie-Virologie-Hygiène (45.01)

Pharmacologie Fondamentale (48.03)

Biochimie et biologie moléculaire (44.01)

Biophysique et Médecine Nucléaire (43.01)

Bactérilogie-virologie ( 45.01)

Cytologie et Histologie (42.02)

Bactériologie-virologie ; Hygiène hospitalière (45.01)

Physiologie (44.02)

Chirurgie Viscérale ( 52-02)

Cardiologie (51.02)

Dermatologie (50.03)

Anatomie et Cytologie pathologiques (42.03)

Biochimie et Biologie moléculaire (44.01)

Chirurgie maxillo-faciale et stomatologie (55.03)

Cardiologie (51.02)

Épidémiologie Économie de la Santé et Prévention (46.01)

Pédopsychiatrie ( 49-04)

Hématologie et Transfusion (47.01)

Pédiatrie (54.01)

PROFESSEUR DES UNIVERSITÉS

M. DARMON David

Médecine Générale (53.03) 
MAITRE DE CONFÉRENCES DES UNIVERSITÉS

Mme GROS Auriane Orthophonie (69)

PROFESSEURS AGRÉGÉS

Mme LANDI Rebecca

Anglais

PRATICIEN HOSPITALIER

UNIVERSITAIRE

M. DURAND Matthieu

Urologie (52.04)

M. SICARD Antoine

Néphrologie (52-03)

\section{PROFESSEURS ASSOCIÉS}

M. GARDON Gilles

Médecine Générale (53.03)

Mme MONNIER Brigitte

Médecine Générale (53.03)

\section{ASSOCIÉS}

MAITRES DE CONFÉRENCES

$\begin{array}{ll}\text { Mme } & \text { CASTA Céline } \\ \text { M. } & \text { GASPERINI Fabrice } \\ \text { M. } & \text { HOGU Nicolas }\end{array}$

Médecine Générale (53.03)

Médecine Générale (53.03)

Médecine Générale (53.03)

\section{Professeurs Honoraires}

M. AMIEL Jean

M. GÉRARD Jean-Pierre

M. ALBERTINI Marc

M. GIBELIN Pierre

M. BALAS Daniel

M. GILLET Jean-Yves

M. BATT Michel

M. GRELLIER Patrick

M. BLAIVE Bruno

M. GRIMAUD Dominique

M. BOQUET Patrice

M. HOFLIGER Philippe

M. BOURGEON André

$M$. JOURDAN Jacques

M. BOUTTÉ Patrick

M. LAMBERT Jean-Claude

M. BRUNETON Jean-Noël

M. LAZDUNSKI Michel

Mme BUSSIERE Françoise

M. CAMOUS Jean-Pierre

M. CANIVET Bertrand

M. CASSUTO Jill-patrice

M. LEFEBVRE Jean-Claude

M. LE FICHOUX Yves

Mme LEBRETON Elisabeth

M. CHATEL Marcel

M. MARIANI Roger

M. MASSEYEFF René

M. COUSSEMENT Alain

Mme CRENESSE Dominique

M. MATTEI Mathieu

M. MOUIEL Jean

M. DARCOURT GuY

M. DELLAMONICA Pierre

Mme MYQUEL Martine

M. ORTONNE Jean-Paul

M. DELMONT Jean

M. PRINGUEY Dominique

M. DEMARD François

M. SANTINI Joseph

M. DESNUELLE Claude

M. SAUTRON Jean Baptiste

M. DOLISI Claude

M. SCHNEIDER Maurice

Mme EULLER-ZIEGLER Liana

M. THYSS Antoine 
M. FENICHEL Patrick

M. FUZIBET Jean-Gabriel

M. FRANCO Alain

M. FREYCHET Pierre

M. GASTAUD Pierre

\section{M.C.U. Honoraires}

M. ARNOLD Jacques

M. BASTERIS Bernard

M. BENOLIEL José

Mlle CHICHMANIAN Rose-Marie

Mme DONZEAU Michèle

M. EMILIOZZI Roméo

M. FRANKEN Philippe

M. GASTAUD Marcel
M. TOUBOL Jacques

M. TRAN Dinh Khiem

M. VAN

OBBERGHEN

Emmanuel

M. GIUDICELLI Jean

M. MAGNÉ Jacques

Mme MEMRAN Nadine

M. MENGUAL Raymond

M. PHILIP Patrick

M. POIRÉE Jean-Claude

Mme ROURE Marie-Claire

\section{PROFESSEURS CONVENTIONNÉS DE L'UNIVERSITÉ}

M. BERTRAND François

M. BROCKER Patrice

M. CHEVALLIER Daniel

Mme FOURNIER-MEHOUAS Manuella

M. JAMBOU Patrick

M. LEBOEUF Mathieu

Mme NADEAU Geneviève

M. ODIN Guillaume

$M$. PEYRADE Frédéric

M. PICCARD Bertrand

M. QUARANTA Jean-François
Médecine Interne

Médecine Interne Option Gériatrie

Urologie

Médecine

Réadaptation

Coordination

Physique et

prélèvements

d'organes

gynécologie- obstétrique

uro-gynécologie

Chirurgie maxilo-faciale

Onco-Hématologie

Psychiatrie

Santé Publique 


\section{Remerciements}

Aux membres du jury :

Au Professeur Piche, pour votre disponibilité, votre écoute et vos efforts pour que tous les internes puissent profiter de leur internat.

Au Professeur Hébuterne, tout d'abord pour vos enseignements en $\mathrm{MICl}$ et en Nutrition. Je pense que mes patients n'échapperont pas à la SNG d'alimentation quand elle est nécessaire et éviterons la NP quand elle n'est pas indispensable. Merci également de m'avoir donné l'opportunité d'aller à l'ECCO à Vienne en présentant un cas clinique au concours « $\mathrm{MICl}$ au grand jour ». Pour vos enseignements en endoscopie également et votre patience (j'essaierai de déboucler plus souvent).

A Stéphane, pour avoir accepté de diriger ma thèse. Merci d'avoir répondu à mes questions farfelues, d'avoir relu, corrigé et upgrader moulte fois mon travail et d'avoir survécu à mon orthographe. Merci également pour tes enseignements en nutrition, pour ta bonne humeur et ton sens de la conversation.

Au Pr Vanbiervliet/A Geoffroy. Je vous/te laisse choisir la formule d'usage ou votre/ton prénom. Merci pour tes/vos enseignements en endoscopie, peut-être un peu tardif sur la partie pratique mais j'ai encore 2 ans pour profiter de vos/tes conseils experts. Merci pour la patience que j'ai dû parfois mobiliser.

A Delphine, pour m'avoir supporté pendant 2 semestres, surtout le premier qui n'a pas été évident mais qui m'a aidé à progresser par la suite. Ton humeur régulière et ta douceur ont rendu les choses plus faciles. Merci pour tes enseignements en cancéro et en puéricultures.

\section{A mes Professeurs :}

Au Pr Tran, pour m'avoir transmis une partie de votre rigueur, d'esprit de synthèse et pour m'avoir appris à toujours raisonner sur les faits.

A Rodolphe, merci d'avoir accepté de chapeauter mon mémoire. J'espère garder de mon stage en hépato tes qualités de clinicien et ton approche globale des patients. Je ne devrai plus envoyer de patient édenté à un panorex...

\section{A mes Maîtres :}

A Eve. Je dois reconnaître que le jour où, très jeune interne, j'ai failli perdre un tympan parce que je n'avais pas scotché l'observation dans le dossier d'un patient des soins je n'imaginais pas que j'aurai autant de plaisir à travailler avec toi dans ton service comme interne. Merci pour tes enseignements, notamment en pancréas, en hépato mais aussi en endoscopie (en musique souvent en plus). Mais également pour m'avoir transmis, non sans douleur parfois, ton sens de la rigueur. J'ai également apprécié d'avoir une chef capable de parler de beaucoup d'autres choses que de médecine à l'internat.

A Cécile. Que dire qui ne sera pas réutilisé contre moi pendant les 2 prochaines années ? Jamais aucun chef n'a été aussi proche du titre chef préféré. Quel dommage que tu déploies autant d'effort à m'effleurer les oreilles et à monopoliser l'ordi du SMC à moitié (complètement ?) allongée sur la chaise. Je te concèderai quand même que tu as toujours été d'un soutient infaillible, que ce soit 
quand j'étais jeune interne un peu perdu, pour une demande d'une kystogastro (même si ça n'est jamais assez collecté pour Madame), pour un avis ou conseil ou pour m'aider à finir les entrées au SMC. Tu es également très bon GPS en endoscopie et bon professeur même si Clément explique mieux ;p Et il, faut le reconnaître, tu es assez drôle.

A Anne-Claire, dont le débit de parole n'a d'égal que son sens clinique. Merci d'avoir résisté à l'envie de me tuer lors de mon début d'internat et de la patience que cela a dû exiger. J'ai beaucoup apprécié travailler avec toi, surtout les 2 semestres au 3C. Et oui, on s'ennuierait beaucoup si tu n'étais pas là. D'ailleurs, merci de me rappeler de manière régulière la chance que j'ai de ne jamais avoir à vivre un accouchement.

A Jérôme, pour m'avoir transmis ton intérêt pour les $\mathrm{MICl}$, pour tes enseignements, ta disponibilité à chaque fois que j'ai pu avoir besoin de toi pour un patient et pour tes blagues graveleuses.

A Ophélie, pour avoir été la chef infaillible, omnisciente et exemplaire dont les jeunes internes ont besoin. J'admirerai toujours ton implication et le niveau d'exigence que tu mets dans ton travail.

A Kamel, pour toutes les endoscopies que je t'ai demandées, rajoutées et celles sur lesquelles tu m'as permis de me former.

A Julie Bébénard, pour ta coolitude (pas que vestimentaire), ta bonne humeur et tes enseignements en $\mathrm{MICl}$ et en endo. Merci d'avoir toujours été volontaire pour me filer un coup de main sauf quand il s'agit de demander un KT à GV (même quand je gagne à Pierre-Papier-Ciseaux).

A Clémence, pour m'avoir materné pendant mon semestre d'hépato. Je suis conscient de la chance que j'ai eue.

A Clément, pour avoir été un cointerne et un chef sur qui on peut compter. Merci également d'avoir participé à ma formation en endoscopie malgré tes plannings beaucoup trop chargés et trop riches en polypes de $13 \mathrm{H}$. Et, encore une fois, merci d'expliquer ce que Cécile n'arrive pas à exprimer en endoscopie.

A Audrey, pour avoir été une chef des internes organisée, efficace et solidaire et d'avoir gardé ces qualités une fois chef. Également pour ta participation au planning de service sur ce semestre. Merci aussi d'être moins râleuse que Clément ;p

A Dan. Merci d'être un chef disponible et aidant. Ton calme et ton humeur régulière sont également très appréciables.

A ceux qui ont contribué à ma thèse :

A Amel Chaarana et au Dr Mariné Barjoan, pour avoir réalisé les analyses statistiques, supporté les moultes demandes supplémentaires et supportés mon ignorance en matière de langage excel...

A mes co-internes,

A Juliette, ma moitiée. Merci de m'avoir épaulé ces 4 années. Merci d'avoir toujours été à l'écoute (surtout après les trans). Tu as été une co-interne fiable et solidaire. Efficace aussi, parce qu'avec toi « les chiens ne pissent pas après les roues ». Merci pour ton grain de folie, ta franchise et pour les expressions dont toi seule a le secret ${ }^{\wedge} \wedge$ 
A Maude, ma varoise préférée. Merci de m'avoir materné mon premier semestre où j'étais perdu. J'ai toujours pu compter sur toi par la suite. Merci aussi de m'avoir fait rire pendant ces 4 ans, l'Archet est beaucoup moins fun sans toi.

A Doriane, pour avoir été une si bonne surprise. Tu es notre ptit coup de cœur (pas au sens hépatologique du terme) à tous. Efficace, dynamique, solidaire et fun. Un ptit peu peste aussi, juste ce qu'il faut pour être une super co-interne et future co-chef.

A Edouard, pour avoir toujours eu le bon mot pour nous faire rire. On jurerait que les bordelais sont sympathiques. Merci d'avoir toujours été un super co-interne, les parisiens ont beaucoup de chance de t'avoir.

A Morgane, ma compatriote (\#sudouest). Merci pour d'avoir été une co-interne de qualité depuis que tu es arrivé. Merci pour ta bonne humeur, ta fraîcheur et ton humour.

A Léa et Marie, mes deux perches parisiennes. En dépit de vos tendances instagrameuses bohèmes chics je suis rassuré de savoir quelles super internes j'aurais quand je serai chef. Bon courage à vous pour me supporte. Si je prends trop le melon vous aurai le droit de réclamer un gâteau (j'espère que Bébénard ne lie pas ça).

A Thomas, pour m'avoir supporté moi et mes plannings.

A Anais, mon bébé déjà si grand. Avant que tu arrives à l'USN, je n'imaginais pas pouvoir compter sur un interne en premier semestre aussi rapidement. Tu es vite devenue une cointerne fiable et efficace. J'apprécie ta gentillesse, ne laisse pas les gens en abuser (surtout les chir et les urgentistes).

Aux internes avec qui j'ai cohabité à l'internat et à la vill|llla

Aux équipes soignantes (désolé d'avance si j'en oublie mais il y en a tellement) :

Du SMC : A Elisabeth et Jos' qui m'ont adopté les premières. A Guyard, infirmière infaillible mais beaucoup trop secrète (balance les tes potins!). A la belle Carine. A celles qui m'ont supportée dès le 3C, Céline, Val' et Floriane. Aux AS de choc : Laurent, Samira, Véro et Sarah. Aux "nouveaux " plus si nouveaux que ça : Olivier, Lamia, Lyna, Thierry. A JJ, le chef de la tribu.

Du 4B enfin 4a, enfin le 7810 quoi ! Aux reines mères Ariel et Alex. Aux autres « veilles " Aude, Roukia, Maeva, Julie, Caro et Stef (ma quasi-sœur). Aux nouveaux, Nathan, Aurelia, Olivia, Fatima et Guillaume. A Paolo, Remy, Cindy et Franck. Aux AS de nuit : celle au grand cœur (Lolo) et celle au franc parler (Delphine). A Kanhnika (je l'ai bien écrit tu vois).

Du 3C : Sandrine, Maude, Aurélie, Nolwen, Claire, Ingrid, Romane, Eva, Lisa, Laurence, Loraine, Blandine, Muriel, Sandy, Marie et les nouveaux. A l'admirable Miss Nail !

De l'hépato : à l'infirmière en chef Gorette, Sabino, Juliette, Vanessa, Véro de jour, Véro de nuit, Sandrine, Madly, Marie, Audrey, Olivier et Mme Boomgaert. C'est toujours un plaisir de travailler avec une équipe aussi soudée que la vôtre.

De I'USN : Nathou, Laura, Eva, Rabeb,Najoue, Nadège, Sabrina et la douce Valentina. II en faut de la patience pour supporter vos patient.

De l'endoscopie : Mathilde, Elodie, Alex, Giogiana, Marion et Jean Yves.

De I'HDJ : Noëlle, Alicia, Marie-Pierre, Laure et Audrey. 
Aux super-diets :

La plus folle (Mégane), la plus belle (Evelyne) et celle qui rattrape le niveau des 2 autres (Isa) ;

Aux supers secrétaires :

A Saaaaandrine, la plus vieille interne du 4b. A Léticia et Sylvie, mes commères préférées. A Roselyne, Graciella et Leila. A Flo et Bribri, qui ont du supporté d'entendre beaucoup de bêtise venant du bureau d'à côté. A Christine, Christelle et Mégane, toujours très accueillantes quand je repasse au 3C. A Virginie et Domi, pour m'avoir casé tant d'examens.

A ma famille et aux amis :

A mes parents, c'est de vous que tout part! Merci de m'avoir toujours soutenu, encouragé, conseillé et motivé. J'ai de la chance d'avoir des parents comme vous qui m'aide encore autant dans ma vie d'adulte.

A Julien, qui a toujours été là, et ce depuis le placenta! Je suis content de voir que même malgré la distance nous sommes restés proche et solidaire l'un de l'autre.

A Alexiane, ma grande sœur, qui est passée en éclaireur pour débroussailler la route qui mène de la P1 à la thèse, d'avoir été une maman de substitution au début de notre vie étudiante.

A Matthieu, avec qui j'ai la chance de partager ma vie. De me supporter et de s'occuper de moi. D'être à l'écoute (quoique j'en dise parfois). D'avoir été d'une aussi grande aide pour ce travail (aide psychologique ET méthodologique). Il faudrait surement une thèse dédiée pour te remercier...

A Marc, Françou, Thomas et PJ, pour me faire rire à chaque fois qu'on à l'occasion de se voir. Même si c'est plus rare maintenant qu'on habite loin les uns des autres c'est toujours un plaisir. A Raphael et Camille, pour être des enfants aussi adorables.

A Caro, Sandra et Thomas, pour être des pièces rapportées d'exception !

A Emmanuel et Richard. Quelqu'un vous aura cité dans une thèse ! Merci d'être d'aussi bonne compagnie, de m'avoir appris le mot «tute " et d'être d'un aussi grand soutient pour Matthieu (et avec moi il en bien besoin).

A Rebelle... C'est bon Cécile je déconne. Je l'ai remerciée de vive voix elle ne sait pas encore lire. 


\section{Résumé}

Introduction: L'IFALD (Intestinal Failure Associated Liver Disease) désigne les atteintes hépatiques multifactorielles observées chez des patients souffrant d'insuffisance intestinale sous nutrition parentérale (NP). Si l'IFALD est mieux connue, Il existe peu de littérature sur les anomalies du bilan hépatique $(A B H)$ chez les patients sous NP d'introduction récente.

Patients et méthodes: L'objectif de notre étude était de déterminer la prévalence et I'incidence des $\mathrm{ABH}$ chez des patients sous NP, ainsi que les facteurs associés à la survenue de ces anomalies. Tous les patients hospitalisés dans les services d'Hépato-gastroentérologie et Nutrition du Centre Hospitalier Universitaire de Nice sous NP depuis plus 7 jours et moins de 3 mois ont été inclus de manière consécutive de mai 2019 à mai 2020. Nous avons collecté les données anthropométriques, l'état nutritionnel, les antécédents et pathologies évolutives des patients, la prise de traitements hépatotoxiques et les modalités d'administration de la nutrition parentérale. Le bilan biologique du jour de l'inclusion était recueilli ainsi que, si disponible, le dernier bilan réalisé la semaine précédant I'introduction de la NP. Nous avons sélectionné et comparé six critères biologiques déjà utilisées dans la littérature pour l'IFALD.

Résultats : 82 malades ont été inclus et l'incidence des $A B H$ a pu être évaluée chez 70 d'entre eux. La prévalence des ABH avant introduction de la NP variait de 10,7 \% à 47,1\% et était, pour presque chaque critère, significativement associée à des pathologies hépato-bilio-pancréatiques. La prévalence médiane des $A B H$ après introduction de la NP était de $42,7 \%$ (IQ = 39,0 - 56,1 \%). La prévalence n'augmentait pas de manière significative après introduction de la NP excepté selon le critère stéatose $(10,1 \%$ vs. 59,8 \%, p < 0,01). L'incidence des $A B H$ dans notre étude variait de $4,1 \%$ à $61,3 \%$ selon le critère biologique choisi, avec une incidence médiane à $28,9 \%(\mathrm{IQ}=23,4-34,0 \%)$. Les cas incidents selon I'un des critères de cholestase avaient un IMC significativement plus bas $18,3 \pm 4,5$ vs. $23,2 \pm 6,1$ $\mathrm{kg} / \mathrm{m}^{2}$ ) et répondaient à la définition HAS de la dénutrition sévère $(58,3 \% \mathrm{vs.} 20,0 \%)$. Les cas incidents selon le critère de cytolyse avaient davantage d'insuffisance intestinale $(66.7 \%$ vs. $35.3 \%, p=0,04)$.

Conclusion : La NP moderne ne parait pas être significativement associée à la survenue d'ABH précoces. La découverte d'ABH en début de NP doit orienter en premier lieu vers une pathologie hépato-bilio-pancréatique, mais doit aussi faire évoquer un syndrome de renutrition chez un malade (sévèrement) dénutri. 


\section{Table des matières}

Liste des enseignants au 1er septembre 2020 à la Faculté de Médecine de Nice.......3

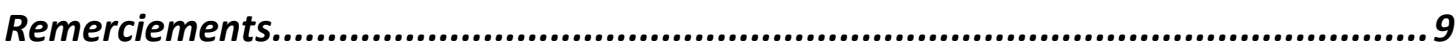

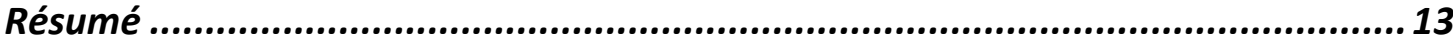

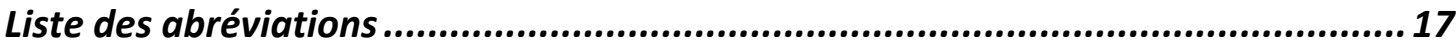

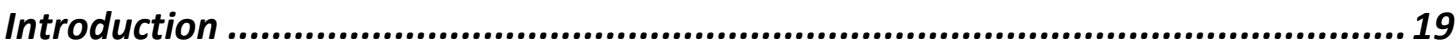

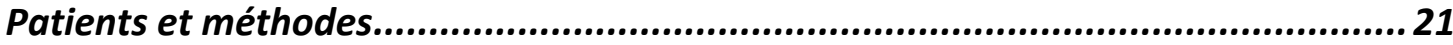

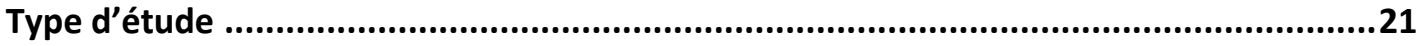

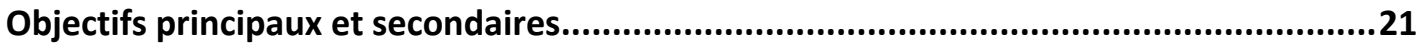

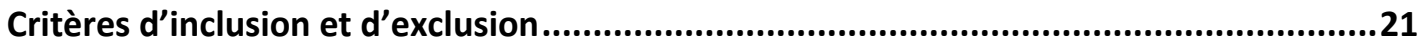

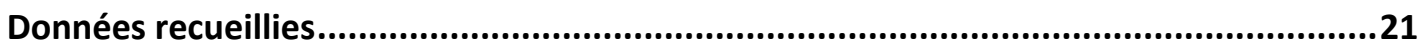

Modalités d'administration de la nutrition parentérale ..............................................22

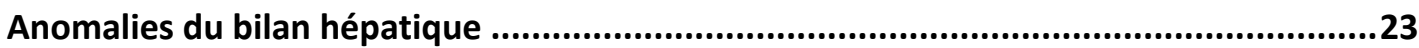

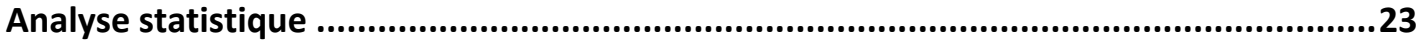

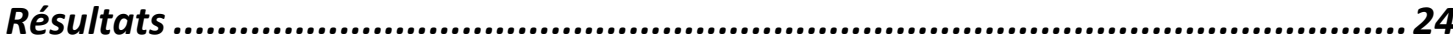

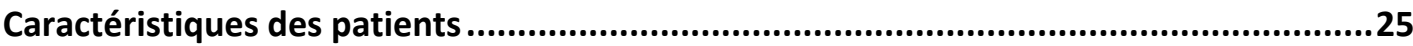

Prévalence des anomalies du bilan hépatique avant introduction de la nutrition

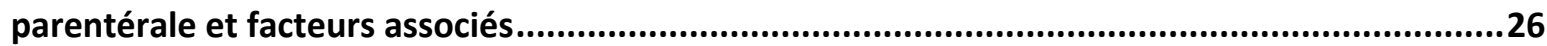

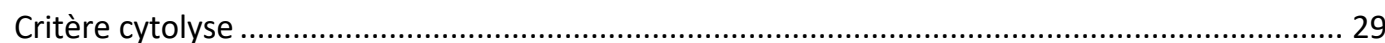

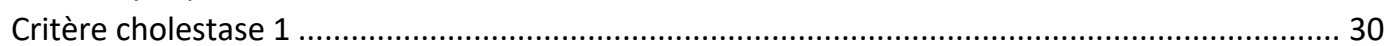

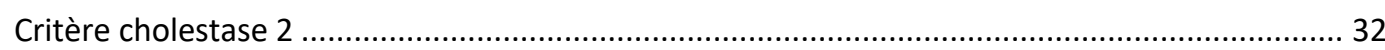

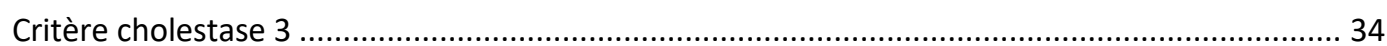

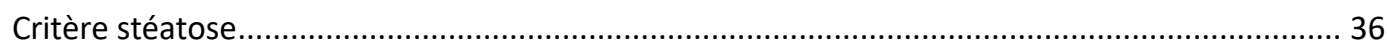

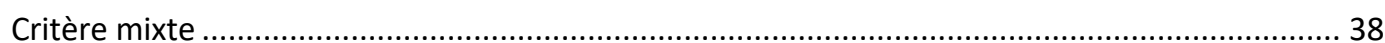

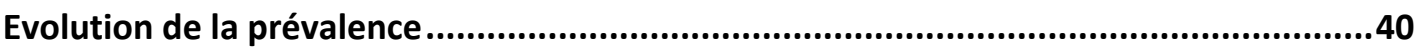

Incidence des anomalies du bilan hépatique ....................................................................42

Facteurs associés aux anomalies du bilan hépatiques incidentes ................................44

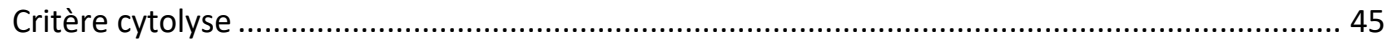

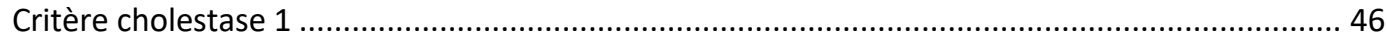

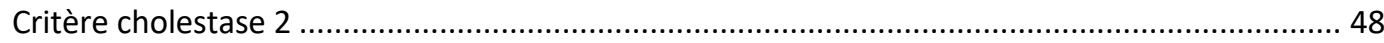

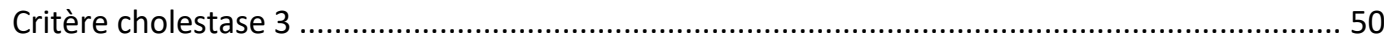

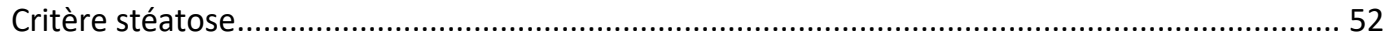

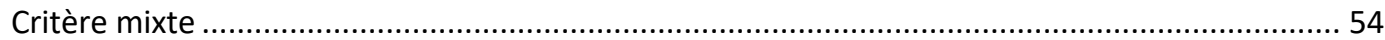

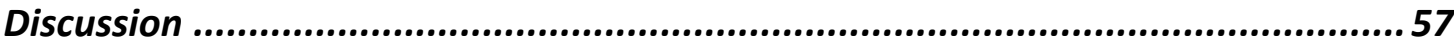

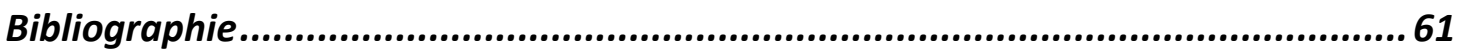

Serment d'Hippocrate.....................................................................................6 63 


\section{Liste des abréviations}

$A B H$ : anomalie(s) biologique(s) hépatique(s)

ALAT : alanine aminotransférase

ASAT : aspartate aminotransférase

g : gramme

GGT : gamma glutamyltranspeptidase

HAS : Haute Autorité de Santé

IC $95 \%$ : intervalle de confiance à $95 \%$

IFALD : Intestinal Failure Associated Liver Disease

IMC : Indice de masse corporelle

$\mathrm{IQ}$ : intervalle interquartile

j : jour

kcal : kilocalorie

$\mathrm{kg}$ : kilogramme

$\mathrm{m}^{2}:$ mètre carré

$\mathrm{MICl}$ : maladie inflammatoire chronique de l'intestin

$n$ : effectif

$\mathrm{N}$ : norme

$\mathrm{N}^{\circ}$ : numéro

OR : Odds Ratio

NP : nutrition parentérale

PAL : phosphatase alcaline

PNALD : Parenteral Nutrition Associated Liver Disease

TP : Taux de prothrombine 


\section{Introduction}

La nutrition parentérale (NP) correspond à l'administration intraveineuse de nutriments, court-circuitant ingestion, digestion et absorption intestinale. L'indication princeps de la NP est l'insuffisance intestinale (1). Cependant, elle est souvent utilisée dans d'autres situations de dénutrition patente ou latente, lorsque la nutrition entérale n'est pas tolérée ou d'utilisation plus complexe.

L'insuffisance intestinale est définie par une incapacité de l'intestin à assurer une absorption suffisante des macronutriments, de l'eau et des électrolytes nécessitant une supplémentation nutritionnelle intraveineuse pour assurer la santé et/ou la croissance (2-4). Elle peut être aiguë ou chronique, réversible ou non selon la quantité d'intestin et de colon fonctionnels en continuité. Les étiologies les plus fréquemment retrouvées sont le syndrome du grêle court, les fistules, les $\mathrm{MICl}$, l'iléus, les troubles de la motricité intestinale, le grêle radique et les néoplasies du tube digestif $(2,4)$. Le syndrome du grêle court - défini par une réduction du grêle en continuité en deçà de $200 \mathrm{~cm}(3,5)$ - constitue la cause la plus fréquente d'insuffisance intestinale. Quelle que soit la cause de l'insuffisance intestinale, outre le traitement étiologique, la prise en charge repose principalement sur le support nutritionnel parentéral (1-4).

Bien que nécessaire sur le plan nutritionnel, la NP expose pourtant à des risques non négligeables et potentiellement mortels : infection et thrombose de cathéter, troubles du métabolisme phosphocalcique, déséquilibre du diabète et atteintes hépatiques allant d'une perturbation isolée des paramètres biologiques hépatiques à la stéatose et la cholestase histologique voire jusqu'à la cirrhose $(2,4)$. Une étude rétrospective multicentrique menée sur 472 patients a estimé un taux de décès à 5 ans chez les patients sous nutrition parentérale ans à $23 \%$. Les décès étaient majoritairement dus à la pathologie digestive sous-jacente ou aux comorbidités, plus importantes avec l'avancée en âge et rarement imputables à la nutrition parentérale (2).

L'Intestinal Failure Associated Liver Disease ou IFALD désigne les atteintes hépatiques propres aux patients souffrant d'insuffisance intestinale sous nutrition parentérale. Le nom de PNALD ou Parenteral Nutrition Associated Liver Disease est de moins en moins utilisé car les anomalies hépatiques constatées ne seraient pas uniquement imputables à la nutrition parentérale mais à d'autres facteurs liés directement ou indirectement à l'insuffisance intestinale (sepsis, insuffisance des ingesta, malabsorption...) $(4,6,7)$. La prévalence est difficile à évaluer et varie de 7 à $65 \%$ selon les critères diagnostiques choisis dans les études, les durées de suivi et le nombre de patients (8-10)

Le diagnostic d'IFALD repose sur un faisceau d'arguments cliniques, biologiques, radiologiques et parfois histologiques $(6,11,12)$. Plusieurs valeurs seuils biologiques ont été proposées. Cavicchi et al. ont classé les patients porteurs d'IFALD en cas d'élévation au-delà de 1,5 fois la normale à 6 mois de l'introduction de la nutrition parentérale de 2 des 3 marqueurs de cholestase suivants : gammaglutamyl transpeptidase (GGT), phosphatases alcalines (PAL) et bilirubine conjuguée (9). Luman et Shaffer ont défini l'existence d'une IFALD en cas d'élévation d'un des marqueurs de la fonction hépatique au-delà de $1.5 \mathrm{~N} 6$ mois après introduction de la nutrition parentérale parmi : ALAT, PAL ou la bilirubine (13). Il existe d'autres définitions basées sur le rapport ASAT/ALAT, la stéatose échographique et les marqueurs non invasifs de fibrose $(7,8)$. La place du FibroScan ${ }^{\odot}$ est difficile à évaluer et une élévation des valeurs mesurées par ce dernier pourrait être davantage corrélée à la cholestase qu'à la fibrose (14). II n'est pas recommandé de réaliser de manière systématique une biopsie hépatique pour poser le diagnostic $(6,15)$. Quand elle est réalisée, l'anatomopathologie montre principalement des lésions de cholestase mais également de la stéatose micro et macrovésiculaire à prédominance péri-portale. La stéatohépatite est rarement retrouvée et la fibrose, présente dans les 
formes évoluées, est généralement discontinue en "pièce de puzzle " (7). II n'existe donc pas de définition standardisée de l'IFALD, si bien qu'elle doit être suspectée en cas d'anomalie hépatique biologique et/ou radiologique et/ou histologique et après avoir éliminé les diagnostics différentiels (hépatites virales, auto-immunes, toxiques, obstacle biliaire...) $(6,11,12,15)$.

La physiopathologie est complexe et multifactorielle. La carence chronique en choline, en carnitine et en taurine, l'administration d'aluminium et de phytostérols contenus dans la nutrition parentérale ainsi que l'excès de glucose et de lipides, particulièrement l'administration d'émulsions lipidiques à base d'huile de soja riches en omega-6, seraient associées aux anomalies hépatiques $(4,6,7,11,12,15)$. Les patients présentant un grêle court et/ou une inflammation intestinale sont plus à risque de développer une cholestase chronique et une fibrose hépatique $(6,12,15)$. Le microbiote intestinal résident (colique) ou ectopique (colonisation bactérienne chronique du grêle) pourraient également jouer un rôle $(11,15,16)$. Le sepsis est un facteur de risque indépendant et pourrait multiplier par trois le risque d'IFALD $(11,12)$.

L'IFALD peut significativement altérer la fonction hépatique et évoluer vers la cirrhose $(6,12)$. Dans l'étude de Cavicchi et al, certes ancienne, $50 \%$ des patients atteints d'IFALD sous nutrition parentérale depuis en moyenne 6 ans ont développé une hépatopathie évoluée. Cette dernière était définie par la présence d'une hypertension portale, d'une fibrose péri-portale ou d'une cirrhose à la biopsie, d'un ictère, d'une ascite, d'une encéphalopathie hépatique, d'une rupture de varice œsophagienne ou d'une altération de la fonction hépatique (Facteur $V<50 \%$ ). $34 \%$ de ces patients sont décédés de leur hépatopathie après 4 ans de nutrition parentérale (9) .

La prévention et le traitement de l'IFALD reposent, lorsque cela est possible, sur l'éviction des hépatotoxiques (alcool, médicament...), l'administration cyclique de la nutrition parentérale, la remise en continuité digestive notamment colique, la recherche et le traitement d'un sepsis associé, le maintien d'un apport oral ou entéral, le traitement de la pullulation microbienne, la maitrise des apports (éviter la « surnutrition »), la limitation des apport en lipides issus d'huile de soja à $1 \mathrm{~g} / \mathrm{kg} / \mathrm{jour}$ et la réduction du rapport en acide gras polyinsaturés omega-6/omega-3, en favorisant les émulsions lipidiques à base d'huile de poisson (1,4,6,11,12,15,17-19). Pour les fibroses évoluées et/ou cirrhoses une double transplantation (hépatique et intestinale) peut être envisagée $(8,11,12)$.

Si les atteintes hépatiques chez les patients sous nutrition parentérale au long cours ont bien été décrites, en revanche il existe peu de littérature sur la survenue d'anomalies du bilan hépatique chez les patients sous NP d'introduction plus récente. L'objectif de notre étude était de décrire ces anomalies et de rechercher les potentiels facteurs associés. 


\section{Patients et méthodes}

\section{Type d'étude}

Il s'agit d'une étude de cohorte prospective menée sur les patients hospitalisés bénéficiant de I'introduction récente d'une NP.

\section{Objectifs principaux et secondaires}

L'objectif de notre étude était de déterminer la prévalence et l'incidence des anomalies biologiques hépatiques des patients sous nutrition parentérale ainsi que les facteurs associés à la survenue de ces anomalies.

\section{Critères d'inclusion et d'exclusion}

Tous les patients hospitalisés dans les services d'Hépato-gastroentérologie et Nutrition du Centre Hospitalier Universitaire de Nice sous nutrition parentérale depuis plus 7 jours et moins de 3 mois ont été inclus de manière consécutive de mai 2019 à mai 2020. Étaient exclus les patients pour lesquels nous n'avions de pas de bilan hépatique disponible.

\section{Données recueillies}

Nous avons collecté les données anthropométriques (âge, sexe, poids, taille, indice de masse corporelle, poids à $M-1$, poids à $M-6$ ), les antécédents et pathologies évolutives des patients tels que les antécédents médicaux, les antécédents chirurgicaux digestifs, l'état nutritionnel, la présence d'une insuffisance intestinale et son étiologie, la longueur de grêle restant pour les grêles courts, la longueur de colon restant (20), les pathologies lithiasiques, les pathologies hépatobiliaires (obstacle biliaire, métastase hépatique, hépatite aigue ou chronique, abcès hépatique...), l'existence d'un sepsis c'est-àdire une infection bactérienne évolutive (angiocholite, abcès intra abdominaux, infection de cathéter, infection urinaire, infection pulmonaire...), la prise de traitements hépatotoxiques et les modalités d'administration de la nutrition parentérale (nombre de jours depuis l'introduction, administration exclusive, quantité de calories, glucides, lipides et protéines).

L'état nutritionnel était apprécié selon les critères HAS (Haute Autorité de Santé) basés sur le pourcentage de perte de poids à 1 et 6 mois, I'IMC (Indice de Masse corporelle) et l'albuminémie (21).

Le bilan biologique du jour de l'inclusion était recueilli ainsi que le dernier bilan réalisé la semaine précédant l'introduction de la nutrition parentérale. Les paramètres biologiques suivants étaient relevés: aspartate aminotransférase (ASAT), alanine aminotransférase (ALAT), gamma glutamyltranspeptidase (GGT), phosphatases alcalines (PAL), bilirubine totale et conjuguée, albumine, taux de prothrombine (TP). 


\begin{tabular}{|c|c|}
\hline $\begin{array}{l}\text { Nom du critère } \\
\text { biologique }\end{array}$ & Paramètres biologiques \\
\hline Cytolyse & ALAT et/ou ASAT $\geq 1,5 \mathrm{~N}$ \\
\hline Cholestase $1^{(9)}$ & $\begin{aligned} \underline{2} \text { paramètres parmi : } \\
\text { - } \mathrm{PAL} \geq 1,5 \mathrm{~N} \\
\text { - } \mathrm{GGT} \geq 1,5 \mathrm{~N} \\
\text { - } \text { Bilirubine conjuguée } \geq 1,5 \mathrm{~N}\end{aligned}$ \\
\hline Cholestase $2^{(5)}$ & Bilirubine conjuguée $>0,3 \mathrm{mg} / \mathrm{dl}$ \\
\hline Cholestase $3^{(5)}$ & $\begin{array}{l}\text { Bilirubine totale }>1 \mathrm{mg} / \mathrm{dl} \\
\frac{\text { ET }}{\text { Bilirubine conjuguée }>0,3 \mathrm{mg} / \mathrm{dl}}\end{array}$ \\
\hline Stéatose ${ }^{(8)}$ & ASAT/ALAT $<1$ si ASAT et ALAT $>N$ \\
\hline Mixte ${ }^{(13)}$ & $\begin{array}{l}1 \text { paramètre parmi : } \\
\text { - } \quad \mathrm{PAL} \geq 1,5 \mathrm{~N} \\
\text { - } \mathrm{ALAT} \geq 1,5 \mathrm{~N} \\
\text { - } \text { Bilirubine } \geq 1,5 \mathrm{~N}\end{array}$ \\
\hline
\end{tabular}

Figure 1-Critères biologiques proposés dans I'IFALD 


\section{Modalités d’administration de la nutrition parentérale}

La nutrition parentérale était administrée sur un abord veineux central. La quasi-totalité des patients était sous Olimel N7E ${ }^{\circledR}$ (Baxter, Guyancourt, France), administrée de manière cyclique ou continue selon la tolérance glycémique. Une ampoule de Cernevit ${ }^{\circledR}$ (Baxter, Guyancourt, France) et de Nutryelt ${ }^{\circledR}$ (Aguettant, Lyon, France) étaient administrées quotidiennement dans la poche de NP. Un seul patient était sous poche selon la formule P3T-SMOF ${ }^{\circledR}$-Dipeptiven ${ }^{\circledR} 150 \mathrm{ml} 2706$ (Fresenius-Kabi, Sèvres, France), fabriquée par le service de pharmacotechnie de la pharmacie à usage interne du CHU de Nice.

\section{Anomalies du bilan hépatique}

Étant donné qu'il n'existe pas de critère biologique unique et consensuel pour parler d'IFALD nous avons sélectionné et comparé plusieurs valeurs seuils biologiques déjà utilisées dans la littérature. Un critère biologique était basé sur la cytolyse. Trois critères biologiques étaient basés sur la cholestase. Un critère était basé sur un indice de stéatose. Le dernier critère était mixte. (Figure 1).

L'incidence des anomalies selon chaque critère a été calculée en définissant comme anomalie incidente toute anomalie du bilan hépatique $(A B H)$ à l'inclusion répondant au critère biologique choisi et non présente sur le bilan avant introduction de la nutrition parentérale.

\section{Analyse statistique}

Le test de $X^{2}$ était utilisé pour comparer les fréquences pour des groupes indépendants. Lorsque les groupes étaient appariés (comparaison avant/après), un test de McNemar avec correction de Yates était utilisé. Dans les deux cas le degré de liberté était de 1.

Lorsque l'analyse univariée mettait en évidence une différence significative une analyse multivariée par modèle de régression logistique était réalisée. Lorsque l'analyse comparative multivariée était significative un Odds ratio (OR) était calculé.

Le seuil de significativité était fixé à $p \leq 0,05$.

Toutes les analyses ont été réalisées en utilisant le logiciel SAS ${ }^{\circledR}$. 

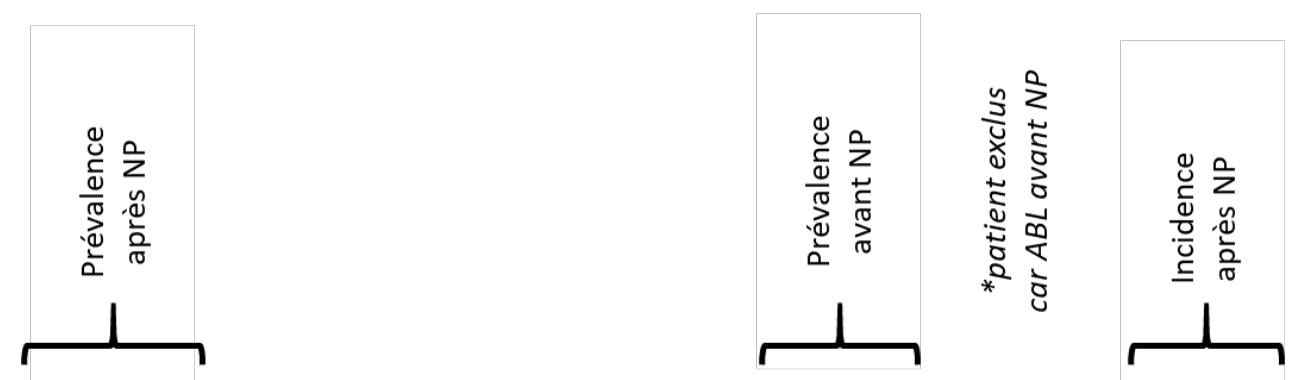

을

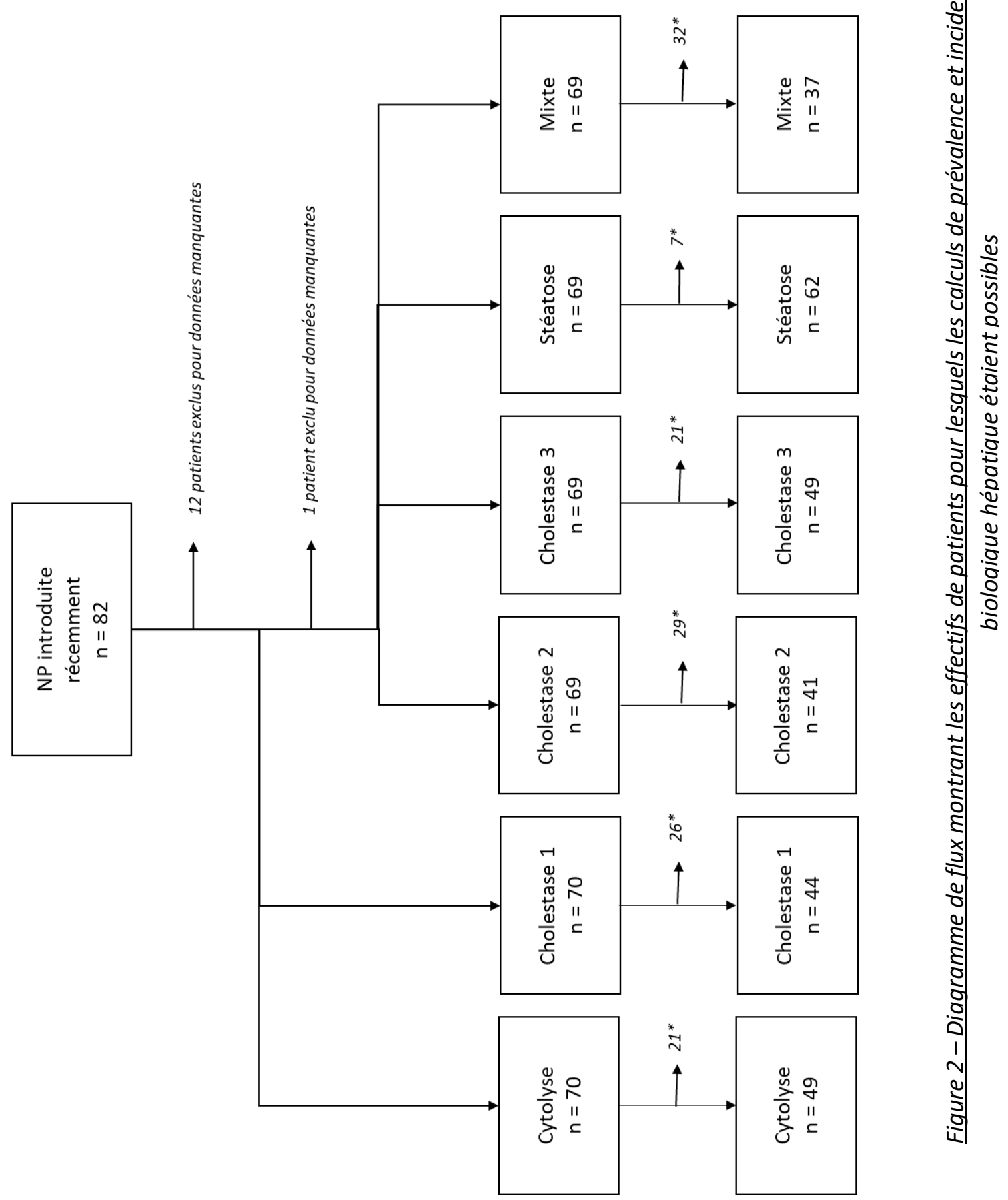




\section{Résultats}

\section{Caractéristiques des patients}

Au total, 82 patients ont été inclus dans l'étude. Nous avons pu déterminer la prévalence des anomalies du bilan hépatique $(A B H)$ à l'inclusion pour 82 patients. Nous avons pu déterminer la prévalence des $A B H$ avant l'introduction de la NP pour 70 patients selon les critères biologiques cytolyse et cholestase 1 et pour 69 patients selon les autres critères biologiques. Nous avons par la suite déterminé l'incidence des $\mathrm{ABH}$ selon chaque critère chez les patients dont le bilan biologique n'était pas perturbé avant introduction de la NP. (Figure 2).

La majorité d'entre eux $(63,4 \%)$ étaient des hommes. L'IMC moyen était à $21,9( \pm 5,5) \mathrm{kg} / \mathrm{m}^{2}$. $53,7 \%$ des patients n'étaient pas dénutris, 9,8 \% présentaient une dénutrition modérée et 30,5\% étaient dénutris sévère. Les patients présentaient majoritairement une pathologie digestive bénigne non hépatobiliaire $(37,8 \%)$ et $32,9 \%$ d'entre eux présentaient un cancer. $69,5 \%$ des patients n'avaient pas eu de chirurgie digestive et $13,4 \%$ des patients avaient un antécédent de cholécystectomie. II y avait $53,7 \%$ d'insuffisance intestinale, majoritairement secondaire à un trouble de la motilité $(17,1 \%)$. On dénombrait $14,6 \%$ de grêle court avec une longueur restante de grêle moyenne évaluée à 50 ( \pm 30) $\mathrm{cm}$. 46,3\% des patients présentaient un sepsis, 8,5\% avaient un sepsis d'origine hépatobiliaire. $34,1 \%$ souffraient d'une pathologie hépatobiliaire et $9,8 \%$ avaient une pathologie lithiasique. 54,9\% des patients ne prenaient pas de traitement hépatotoxique. Les apports énergétiques moyens parentéraux étaient de $29,0( \pm 10,0) \mathrm{kcal} / \mathrm{kg} / \mathrm{j}$, dont $3,5( \pm 1,2) \mathrm{g} / \mathrm{kg} / \mathrm{j}$ de glucides, $1,1( \pm 0,3) \mathrm{g} / \mathrm{kg} / \mathrm{j}$ de protéine et $1,0( \pm 0,3) \mathrm{g} / \mathrm{kg} / \mathrm{j}$ de lipide ( $\underline{\text { Tableau } 1})$. 
Tableau 1 - Caractéristiques des 82 patients à l'inclusion

\begin{tabular}{|c|c|}
\hline Age moyen (années) & $61( \pm 15,1)$ \\
\hline $\begin{array}{l}\text { Homme } \\
\text { Femme }\end{array}$ & $\begin{array}{l}52(63,4 \%) \\
30(36,6 \%)\end{array}$ \\
\hline $\begin{array}{l}\mathrm{IMC} \text { moyen }\left(\mathrm{kg} / \mathrm{m}^{2}\right)^{\dagger} \\
\text { Dénutrition sévère selon IMC } \\
\text { Dénutrition modérée selon IMC } \\
\text { Normal } \\
\text { Surpoids } \\
\text { Obésité }\end{array}$ & $\begin{array}{c}21,9( \pm 5,5) \\
17(20.7 \%) \\
10(12.2 \%) \\
32(39.0 \%) \\
11(13.4 \%) \\
5(6.1 \%)\end{array}$ \\
\hline $\begin{array}{l}\text { Dénutrition selon la } \mathrm{HAS}^{\dagger} \\
\text { Modérée } \\
\text { Sévère }\end{array}$ & $\begin{array}{c}41(48,3 \%) \\
8(9,8 \%) \\
25(30,5 \%) \\
\end{array}$ \\
\hline $\begin{array}{l}\text { Pathologie principale } \\
\text { Cancer } \\
\text { Pathologie hépato-bilio-pancréatique bénigne } \\
\text { Autre pathologie digestive } \\
\text { Autre }\end{array}$ & $\begin{array}{l}27(32,9 \%) \\
18(22,0 \%) \\
31(37,8 \%) \\
6(7,3 \%)\end{array}$ \\
\hline $\begin{array}{l}\text { Antécédent de chirurgie du tube digestif } \\
\text { Résection iléale } \\
\text { Résection colique } \\
\text { Résection iléo-colique } \\
\text { Chirurgie sus mésocolique }\end{array}$ & $\begin{array}{c}28(30,5 \%) \\
8(9,8 \%) \\
5(6,1 \%) \\
6(7,3 \%) \\
6(7,3 \%)\end{array}$ \\
\hline Cholécystectomie & $11(13,4 \%)$ \\
\hline $\begin{array}{l}\text { Nombre de jours moyens depuis début de la } \\
\text { nutrition parentérale }\end{array}$ & $20,6( \pm 14,2)$ \\
\hline $\begin{array}{l}\text { Insuffisance intestinale } \\
\text { Grêle court } \\
\text { Fistule intestinale } \\
\text { Trouble moteur } \\
\text { Occlusion } \\
\text { Atteinte muqueuse } \\
\end{array}$ & $\begin{array}{c}4(53,7 \%) \\
12(14,6 \%) \\
3(3,7 \%) \\
14(17,1 \%) \\
13(15,9 \%) \\
2(2,4 \%) \\
\end{array}$ \\
\hline NP exclusive & $19(23,2 \%)$ \\
\hline $\begin{array}{l}\text { Longueur moyenne de grêle restant chez les grêles } \\
\text { courts (mètres) }\end{array}$ & $0,5( \pm 0,3)$ \\
\hline Longueur moyenne de colon restant (\%) & $92 \%$ \\
\hline $\begin{array}{l}\text { Sepsis } \\
\text { D'origine hépato-biliaire } \\
\text { D'autre origine }\end{array}$ & $\begin{array}{l}38(46,3 \%) \\
7(8,5 \%) \\
31(37,8 \%)\end{array}$ \\
\hline $\begin{array}{l}\text { Pathologie hépato-biliaire } \\
\text { Lithiasique } \\
\text { Non lithiasique }\end{array}$ & $\begin{array}{c}28(34,1 \%) \\
8(9,8 \%) \\
20(24,3 \%) \\
\end{array}$ \\
\hline $\begin{array}{c}\text { Traitements hépatotoxiques } \\
\text { Antibiothérapie } \\
\text { Chimiothérapie }\end{array}$ & $\begin{array}{r}37(\mathbf{4 5}, \mathbf{1} \%) \\
26(31,7 \%) \\
11(13,4 \%)\end{array}$ \\
\hline $\begin{array}{l}\text { Apports caloriques parentéraux moyens }(\mathrm{kcal} / \mathrm{kg} / \mathrm{j}) \\
\text { Dont glucides }(\mathrm{g} / \mathrm{kg} / \mathrm{j}) \\
\text { Dont protides }(\mathrm{g} / \mathrm{kg} / \mathrm{j}) \\
\text { Dont lipides }(\mathrm{g} / \mathrm{kg} / \mathrm{j})\end{array}$ & $\begin{array}{r}29,0( \pm 10,0) \\
3,5( \pm 1,2) \\
1,1( \pm 0,3 \\
1,0( \pm 0,3\end{array}$ \\
\hline
\end{tabular}

$\dagger$ : analyse effectuée sur $n=75$ patients (données anthropométriques manquantes) 
Tableau 2-Comparaison des patients avec et sans anomalies du bilan hépatique selon le critère cytolyse, avant introduction de la nutrition parentérale

\begin{tabular}{|c|c|c|c|}
\hline & $\begin{array}{c}\text { Oui } \\
n=21(30 \%)\end{array}$ & $\begin{array}{c}\text { Non } \\
n=49(70 \%)\end{array}$ & $\mathbf{p}$ \\
\hline Age (années) & $57( \pm 12,7)$ & $62( \pm 15,2)$ & 0,2 \\
\hline \multicolumn{4}{|l|}{ Sexe } \\
\hline Homme & $15(71,4 \%)$ & $30(61,2 \%)$ & \multirow[t]{2}{*}{0,4} \\
\hline Femme & $6(28,6 \%)$ & $19(38,8 \%)$ & \\
\hline IMC moyen $\left(\mathrm{kg} / \mathrm{m}^{2}\right)^{\dagger}$ & $20,9( \pm 4,6)$ & $22,1( \pm 5,4)$ & 0,4 \\
\hline Dénutrition sévère selon IMC & $6(30,0 \%)$ & $10(21,7 \%)$ & 0,4 \\
\hline Dénutrition modérée selon IMC & $2(10,0 \%)$ & $7(15,2 \%)$ & 0,5 \\
\hline Normal & $8(40,0 \%)$ & $19(41,3 \%)$ & 0,8 \\
\hline Surpoids & $4(20,0 \%)$ & $6(13,0 \%)$ & 0,4 \\
\hline Obésité & $0(0,0 \%)$ & $4(8,7 \%)$ & 0,3 \\
\hline \multicolumn{4}{|l|}{ 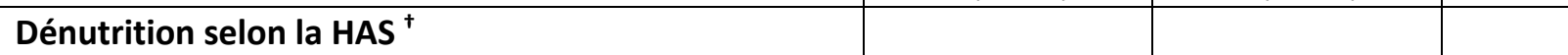 } \\
\hline Modérée & $0(0,0 \%)$ & $6(13,0 \%)$ & 0,09 \\
\hline Sévère & $4(20,0 \%)$ & $17(37,0 \%)$ & 0,1 \\
\hline \multicolumn{4}{|l|}{ Pathologie principale } \\
\hline Cancer & $5(23,8 \%)$ & $20(40,8 \%)$ & 0,07 \\
\hline Pathologie hépato-bilio-pancréatique bénigne & $7(33,3 \%)$ & $9(18,4 \%)$ & 0,5 \\
\hline Autre pathologie digestive & $5(23,8 \%)$ & $18(36,7 \%)$ & 0,1 \\
\hline Autre & $4(19,0 \%)$ & $2(4,1 \%)$ & 0,06 \\
\hline Antécédent de chirurgie du tube digestif & $3(14,3 \%)$ & $15(30,6 \%)$ & \\
\hline Résection iléale & $1(4,8 \%)$ & $4(8,2 \%)$ & 0,5 \\
\hline Résection colique & $1(4,8 \%)$ & $4(8,2 \%)$ & 0,4 \\
\hline Résection iléo-colique & $1(4,8 \%)$ & $2(4,1 \%)$ & 0,9 \\
\hline Chirurgie sus mésocolique & $0(0,0 \%)$ & $5(10,2 \%)$ & 0,9 \\
\hline Antécédent de cholécystectomie & $1(4,8 \%)$ & $7(14,3 \%)$ & 0,2 \\
\hline Insuffisance intestinale & $7(33,3 \%)$ & $27(55,1 \%)$ & 0,09 \\
\hline Grêle court & $1(4,8 \%)$ & $7(14,3 \%)$ & 0,9 \\
\hline Fistule intestinale & $0(0,0 \%)$ & $3(6,1 \%)$ & 0,9 \\
\hline Trouble moteur & $4(4,8 \%)$ & $6(12,2 \%)$ & 0,9 \\
\hline Obstruction & $2(2,4 \%)$ & $10(20,4 \%)$ & 0,9 \\
\hline Atteinte muqueuse & $0(0,0 \%)$ & $1(2,0 \%)$ & 0,9 \\
\hline NP exclusive & $4(19,0 \%)$ & $14(28,6 \%)$ & 0,7 \\
\hline Longueur moyenne de colon restant (\%) & $90 \%( \pm 20 \%)$ & $90 \%( \pm 20 \%)$ & 0,9 \\
\hline Sepsis & $10(47,6 \%)$ & $22(44,9 \%)$ & 0,8 \\
\hline Dont sepsis d'origine hépatobiliaire & $3(14,3 \%)$ & $4(8,2 \%)$ & 0,4 \\
\hline Pathologie hépatobiliaire & $15(71,4 \%)$ & $12(24,5 \%)$ & $<0,001$ \\
\hline Dont pathologie lithiasique & $3(14,3 \%)$ & $4(8,2 \%)$ & 0,4 \\
\hline Traitements hépatotoxiques & $8(38,1 \%)$ & $23(46,9 \%)$ & - \\
\hline Antibiothérapie & $7(33,3 \%)$ & $15(30,6 \%)$ & 0.2 \\
\hline Chimiothérapie & $1(4,8 \%)$ & $8(16,3 \%)$ & 0.3 \\
\hline
\end{tabular}

$\dagger$ : analyse effectuée sur $n=66$ patients (données anthropométriques manquantes) 


\section{Prévalence des anomalies du bilan hépatique avant introduction de la nutrition parentérale et facteurs associés}

\section{Critère cytolyse}

La prévalence des $\mathrm{ABH}$ avant introduction de la NP selon le critère cytolyse était de 30,0\%. Les patients qui perturbaient leur bilan hépatique avaient significativement plus de pathologies hépatobiliaires $(71,4 \%$ vs. $24,5 \%, p<0,001)$ mais ce résultat n'était pas significatif en analyse multivariée. On notait également, sans significativité, une proportion plus importante de pathologies hépato-biliopancréatiques bénignes en pathologie principale (33,3\% vs. 18,4\%, p = 0,06) ( $\underline{\text { Tableau } 2)}$. 
Tableau 3 - Comparaison des patients avec et sans anomalies du bilan hépatique selon le critère cholestase 1, avant introduction de la nutrition parentérale

\begin{tabular}{|c|c|c|c|}
\hline & $\begin{array}{c}\text { Oui } \\
n=26(37,1 \%)\end{array}$ & $\begin{array}{c}\text { Non } \\
n=44(62,9 \%)\end{array}$ & $\mathbf{p}$ \\
\hline Age (années) & $61( \pm 12,9)$ & $61 \pm(15,7)$ & 0,8 \\
\hline $\begin{array}{l}\text { Sexe } \\
\text { Homme } \\
\text { Femme }\end{array}$ & $\begin{array}{l}16(61,5 \%) \\
10(38,5 \%)\end{array}$ & $\begin{array}{l}29(65,9 \%) \\
15(34,1 \%)\end{array}$ & 0,7 \\
\hline $\begin{array}{l}\text { IMC moyen }\left(\mathbf{k g} / \mathrm{m}^{2}\right) \dagger \\
\text { Dénutrition sévère selon IMC } \\
\text { Dénutrition modérée selon IMC } \\
\text { Normal } \\
\text { Surpoids } \\
\text { Obésité }\end{array}$ & $\begin{array}{c}21,4( \pm 3,5) \\
3(12,5 \%) \\
4(16,7 \%) \\
13(54,2 \%) \\
4(16,7 \%) \\
0(0,0 \%)\end{array}$ & $\begin{array}{c}21,9( \pm 6,1) \\
13(31,0 \%) \\
5(11,9 \%) \\
16(38,1 \%) \\
6(14,3 \%) \\
4(9,5 \%)\end{array}$ & $\begin{array}{l}0,7 \\
0,08 \\
0,6 \\
0,3 \\
0,8 \\
0,2 \\
\end{array}$ \\
\hline $\begin{array}{l}\text { Dénutrition selon la HAS † } \\
\text { Modérée } \\
\text { Sévère }\end{array}$ & $\begin{array}{l}1(4,2 \%) \\
5(20,8 \%)\end{array}$ & $\begin{array}{l}5(11,9 \%) \\
16(38,0 \%)\end{array}$ & $\begin{array}{l}0,2 \\
0,1\end{array}$ \\
\hline $\begin{array}{l}\text { Pathologie principale } \\
\text { Cancer } \\
\text { Pathologie hépato-bilio-pancréatique bénigne } \\
\text { Autre pathologie digestive } \\
\text { Autre }\end{array}$ & $\begin{array}{c}11(42,3 \%) \\
8(30,8 \%) \\
6(23,1 \%) \\
1(3,8 \%)\end{array}$ & $\begin{array}{c}14(31,8 \%) \\
8(18,2 \%) \\
17(38,6 \%) \\
5(11,4 \%)\end{array}$ & $\begin{array}{c}- \\
0,2 \\
0,1 \\
0,5\end{array}$ \\
\hline $\begin{array}{l}\text { Antécédent de chirurgie du tube digestif } \\
\text { Résection iléale } \\
\text { Résection colique } \\
\text { Résection iléo-colique } \\
\text { Chirurgie sus mésocolique }\end{array}$ & $\begin{array}{l}5(19,2 \%) \\
1(3,8 \%) \\
1(3,8 \%) \\
1(3,8 \%) \\
2(7,7 \%)\end{array}$ & $\begin{array}{c}30(29,5 \%) \\
4(9,1 \%) \\
4(9,1 \%) \\
2(4,5 \%) \\
3(6,8 \%)\end{array}$ & $\begin{array}{c}- \\
0,3 \\
0,5 \\
0,5 \\
0,8\end{array}$ \\
\hline Antécédent de cholécystectomie & $3(11,8 \%)$ & $5(11,4 \%)$ & 0,9 \\
\hline $\begin{array}{l}\text { Insuffisance intestinale } \\
\text { Grêle court } \\
\text { Fistule intestinale } \\
\text { Trouble moteur } \\
\text { Obstruction } \\
\text { Atteinte muqueuse }\end{array}$ & $\begin{array}{c}10(38,5 \%) \\
2(7,7 \%) \\
0(0,0 \%) \\
4(15,4 \%) \\
4(15,4 \%) \\
0(0,0 \%)\end{array}$ & $\begin{array}{l}24(54,5 \%) \\
6(13,6 \%) \\
3(6,8 \%) \\
6(13,6 \%) \\
8(18,2 \%) \\
1(2,3 \%)\end{array}$ & $\begin{array}{l}0,1 \\
0,5 \\
0,9 \\
0,9 \\
0,6 \\
0,9\end{array}$ \\
\hline NP exclusive & $5(19,2 \%)$ & $13(29,5 \%)$ & 0,3 \\
\hline Longueur moyenne de colon restant (\%) & $90 \%( \pm 20 \%)$ & $90 \%( \pm 20 \%)$ & 0,6 \\
\hline $\begin{array}{l}\text { Sepsis } \\
\text { Dont sepsis d'origine hépatobiliaire }\end{array}$ & $\begin{array}{c}13(50,0 \%) \\
7(26,9 \%) \\
\end{array}$ & $\begin{array}{c}19(43,2 \%) \\
0(0,0 \%) \\
\end{array}$ & $\begin{array}{c}0,5 \\
<0,001 \\
\end{array}$ \\
\hline $\begin{array}{l}\text { Pathologie hépatobiliaire } \\
\text { Dont pathologie lithiasique }\end{array}$ & $\begin{array}{c}19(73,1 \%) \\
4(15,4 \%) \\
\end{array}$ & $\begin{array}{c}36(81,8 \%) \\
3(6,8 \%)\end{array}$ & $\frac{<0,001}{0,2} \mp$ \\
\hline $\begin{array}{c}\text { Traitements hépatotoxiques } \\
\text { Antibiothérapie } \\
\text { Chimiothérapie }\end{array}$ & $\begin{array}{c}15(57,7 \%) \\
10(38,5 \%) \\
5(19,2 \%)\end{array}$ & $\begin{array}{c}16(36,4 \%) \\
12(27,3 \%) \\
4(9,1 \%)\end{array}$ & $\begin{array}{c}0,07 \\
0,1\end{array}$ \\
\hline
\end{tabular}

$\dagger$ : analyse effectuée sur $n=66$ patients (données anthropométriques manquantes)

$\ddagger$ : en analyse multivariée, $\mathrm{OR}=7,7 ; \mathrm{IC}_{95 \%}[2,3-25,7] ; \mathrm{p}<0,001$ 


\section{Critère cholestase 1}

La prévalence des $A B H$ avant introduction de la NP selon le critère cholestase 1 était de 37,1 $\%$. Les patients qui perturbaient leur bilan hépatique avaient significativement moins de pathologies hépato-biliaires $(73,1 \%$ vs. $81,8 \%, p<0,001)$. Ce résultat était également significatif en analyse multivariée $(\mathrm{OR}=7,7$, IC $95 \% 2,3-25,7, \mathrm{p}<0,001)$. On notait également, une proportion plus importante de sepsis d'origine hépato-biliaires $(26,9 \%$ vs. $0,0 \%, p<0,001)$ mais cela n'était pas significatif en multivarié ( $\underline{\text { Tableau } 3 \text { ). }}$. 
Tableau 4-Comparaison des patients avec et sans anomalies du bilan hépatique selon le critère cholestase 2, avant introduction de la nutrition parentérale

\begin{tabular}{|c|c|c|c|}
\hline & $\begin{array}{c}\text { Oui } \\
n=28(40,6 \%)\end{array}$ & $\begin{array}{c}\text { Non } \\
n=41(59,4 \%)\end{array}$ & $\mathbf{p}$ \\
\hline Age (années) & $63( \pm 14,9)$ & $59( \pm 14,6)$ & 0,3 \\
\hline $\begin{array}{l}\text { Homme } \\
\text { Femme }\end{array}$ & $\begin{array}{c}21(75,0 \%) \\
7(25,0 \%)\end{array}$ & $\begin{array}{l}23(56,1 \%) \\
18(43,9 \%)\end{array}$ & 0,1 \\
\hline $\begin{array}{l}\text { IMC moyen }\left(\mathrm{kg} / \mathrm{m}^{2}\right) \dagger \\
\text { Dénutrition sévère selon IMC } \\
\text { Dénutrition modérée selon IMC } \\
\text { Normal } \\
\text { Surpoids } \\
\text { Obésité }\end{array}$ & $\begin{array}{c}21( \pm 4,1) \\
7(26,9 \%) \\
5(19,2 \%) \\
14(53,8 \%) \\
5(17,9 \%) \\
0(0,0 \%) \\
\end{array}$ & $\begin{array}{c}22,1( \pm 5,8) \\
9(23,0 \%) \\
3(7,7 \%) \\
18(46,1 \%) \\
5(12,8 \%) \\
4(10,2 \%) \\
\end{array}$ & $\begin{array}{l}0,5 \\
0,7 \\
0,1 \\
0,1 \\
0,5 \\
0,1\end{array}$ \\
\hline $\begin{array}{c}\text { Dénutrition selon la HAS † } \\
\text { Modérée } \\
\text { Sévère }\end{array}$ & $\begin{array}{c}11(42,3 \%) \\
3(11,5 \%)\end{array}$ & $\begin{array}{c}10(25,6 \%) \\
3(7,7 \%)\end{array}$ & $\begin{array}{l}0,1 \\
0,6\end{array}$ \\
\hline $\begin{array}{l}\text { Pathologie principale } \\
\text { Cancer } \\
\text { Pathologie hépato-bilio-pancréatique bénigne } \\
\text { Autre pathologie digestive } \\
\text { Autre }\end{array}$ & $\begin{array}{c}7(25,0 \%) \\
11(39,3 \%) \\
7(25,0 \%) \\
3(10,7 \%)\end{array}$ & $\begin{array}{c}17(41,5 \%) \\
5(12,2 \%) \\
16(39,0 \%) \\
3(7,3 \%)\end{array}$ & $\begin{array}{c}- \\
0,1 \\
0,03 \\
0,1\end{array}$ \\
\hline $\begin{array}{l}\text { Antécédent de chirurgie du tube digestif } \\
\text { Résection iléale } \\
\text { Résection colique } \\
\text { Résection iléo-colique } \\
\text { Chirurgie sus mésocolique }\end{array}$ & $\begin{array}{l}6(21,4 \%) \\
1(3,6 \%) \\
2(7,1 \%) \\
2(7,1 \%) \\
1(3,6 \%)\end{array}$ & $\begin{array}{c}11(26,8 \%) \\
4(9,8 \%) \\
2(4,9 \%) \\
1(2,4 \%) \\
4(9,8 \%)\end{array}$ & 0,5 \\
\hline Antécédent de cholécystectomie & $1(3,6 \%)$ & $7(17,1 \%)$ & 0,08 \\
\hline $\begin{array}{l}\text { Insuffisance intestinale } \\
\text { Grêle court } \\
\text { Fistule intestinale } \\
\text { Trouble moteur } \\
\text { Obstruction } \\
\text { Atteinte muqueuse }\end{array}$ & $\begin{array}{c}14(50,0 \%) \\
3(10,1 \%) \\
0(0,0 \%) \\
6(21,4 \%) \\
5(17,9 \%) \\
0(0,0 \%)\end{array}$ & $\begin{array}{c}22(53,7 \%) \\
5(12,2 \%) \\
3(7,3 \%) \\
4(9,9 \%) \\
6(14,6 \%) \\
1(2,4 \%)\end{array}$ & $\begin{array}{l}0,7 \\
0,2 \\
0,9 \\
0,9 \\
0,9 \\
0,9\end{array}$ \\
\hline NP exclusive & $4(14,3 \%)$ & $13(31,7 \%)$ & 0,09 \\
\hline Longueur moyenne de colon restant (\%) & $90 \%( \pm 9 \%)$ & $90 \%( \pm 20 \%)$ & 0,2 \\
\hline $\begin{array}{l}\text { Sepsis } \\
\quad \text { Dont sepsis d'origine hépatobiliaire }\end{array}$ & $\begin{array}{l}15(53,6 \%) \\
6(21,4 \%)\end{array}$ & $\begin{array}{c}17(41,5 \%) \\
1(2,4 \%)\end{array}$ & $\begin{array}{c}0,3 \\
0,01\end{array}$ \\
\hline $\begin{array}{l}\text { Pathologie hépatobiliaire } \\
\text { Dont pathologie lithiasique }\end{array}$ & $\begin{array}{c}19(67,9 \%) \\
5(17,9 \%)\end{array}$ & $\begin{array}{l}8(19,5 \%) \\
2(4,9 \%)\end{array}$ & $\frac{<0,001}{0,07} \mp$ \\
\hline $\begin{array}{c}\text { Traitements hépatotoxiques } \\
\text { Antibiothérapie } \\
\text { Chimiothérapie }\end{array}$ & $\begin{array}{c}17(60,7 \%) \\
11(39,3 \%) \\
0(0,0 \%)\end{array}$ & $\begin{array}{c}19(46,3 \%) \\
11(26,8 \%) \\
8(19,5 \%)\end{array}$ & $\begin{array}{c}- \\
0,04 \\
0,9\end{array}$ \\
\hline
\end{tabular}

+ : analyse effectuée sur $\mathrm{n}=66$ patients (données anthropométriques manquantes)

$\ddagger$ : en analyse multivariée, $O R=7,4 ; I C_{95 \%}[1,6-33,97] ; p<0,009$ 


\section{Critère cholestase 2}

La prévalence des ABH avant introduction de la NP selon le critère cholestase 2 était de 40,6 $\%$. Les patients qui perturbaient leur bilan hépatique avaient significativement plus de pathologies hépato-biliaires $(67,9 \%$ vs. $19,5 \%, p<0,001)$ et ce résultat était significatif en analyse multivariée (OR $=7,4$, IC $95 \%$ 1,6 - 33,9, $p=0,009$ ). On notait également une proportion plus importante de cancers en pathologie principale chez les patients sans $A B H$ selon le critère cholestase 2 ( $25,0 \%$ vs. $41,5 \%$, $p$ $=0,05)$ et davantage de sepsis d'origine hépato-biliaire chez les patients avec $\mathrm{ABH}(21,4 \%$ vs. $2,4 \%, \mathrm{p}$ $=0,01)$ mais ces résultats n'étaient pas retrouvés en analyse multivariée. II y avait également significativement moins de patient sous traitements hépatotoxiques chez les patients avec ABH $(60,7$ $\%$ vs. 53,7\%) mais cela n'était pas significatif en analyse multivariée (Tableau 4). 
Tableau 5-Comparaison des patients avec et sans anomalies du bilan hépatique selon le critère cholestase 3, avant introduction de la nutrition parentérale

\begin{tabular}{|c|c|c|c|}
\hline & \\
\hline & $\begin{array}{c}\text { Oui } \\
N=20(29,9 \%)\end{array}$ & $\begin{array}{c}\text { Non } \\
\mathrm{N}=49(71,1 \%)\end{array}$ & $\mathbf{p}$ \\
\hline Age (années) & $60( \pm 15,5)$ & $61( \pm 14,5)$ & 0,8 \\
\hline \multicolumn{4}{|l|}{ Sexe } \\
\hline Homme & 15 (75,0 \%) & 29 (59,2 \%) & 0,2 \\
\hline Femme & $5(25,0 \%)$ & $20(40,8 \%)$ & \\
\hline IMC moyen $\left(\mathrm{kg} / \mathrm{m}^{2}\right)+$ & $22,1( \pm 4,5)$ & $21,7( \pm 5,5)$ & 0,8 \\
\hline Dénutrition sévère selon IMC & $5(27,8 \%)$ & $11(23,4 \%)$ & 0,8 \\
\hline Dénutrition modérée selon IMC & $1(5,6 \%)$ & $7(14,9 \%)$ & 0,4 \\
\hline Normal & $7(38,9 \%)$ & $20(42,6 \%)$ & 0,9 \\
\hline Surpoids & $5(25,0 \%)$ & $5(10,6 \%)$ & 0,1 \\
\hline Obésité & $0(0,0 \%)$ & $4(8,5 \%)$ & 0,1 \\
\hline \multicolumn{4}{|l|}{ Dénutrition selon la HAS † } \\
\hline Modérée & $0(0,0 \%)$ & $7(12,2 \%)$ & 0,1 \\
\hline Sévère & $12(66,7 \%)$ & $34(72,3 \%)$ & 0,9 \\
\hline \multicolumn{4}{|l|}{ Pathologie principale } \\
\hline Cancer & $6(30,0 \%)$ & $18(36,7 \%)$ & - \\
\hline Pathologie hépato-bilio-pancréatique bénigne & $8(40,0 \%)$ & $8(16,3 \%)$ & 0,5 \\
\hline Autre pathologie digestive & $4(20,0 \%)$ & $19(38,8 \%)$ & 0,07 \\
\hline Autre & $2(10,0 \%)$ & $4(8,2 \%)$ & 0,1 \\
\hline Antécédent de chirurgie du tube digestif & $4(20 \%)$ & $13(26,5 \%)$ & - \\
\hline Résection iléale & $0(0,0 \%)$ & $5(10,2 \%)$ & 0,9 \\
\hline Résection colique & $2(10,0 \%)$ & $2(4,1 \%)$ & 0,4 \\
\hline Résection iléo-colique & $1(5,0 \%)$ & $2(4,1 \%)$ & 0,9 \\
\hline Chirurgie sus mésocolique & $1(5,0 \%)$ & $4(8,2 \%)$ & 0,9 \\
\hline Antécédent de cholécystectomie & $20(100,0 \%)$ & $41(83,7 \%)$ & 0,05 \\
\hline Insuffisance intestinale & $7(35,0 \%)$ & $26(53,1 \%)$ & 0,1 \\
\hline Grêle court & $1(5,0 \%)$ & $7(14,3 \%)$ & 0,5 \\
\hline Fistule intestinale & $0(0,0 \%)$ & $3(6,1 \%)$ & 0,2 \\
\hline Trouble moteur & $5(25,0 \%)$ & $5(10,2 \%)$ & 0,9 \\
\hline Obstruction & $1(5,0 \%)$ & $10(20,4 \%)$ & 0,9 \\
\hline Atteinte muqueuse & $0(0,0 \%)$ & $1(2,0 \%)$ & 0,9 \\
\hline NP exclusive & $3(15,0 \%)$ & $14(28,6 \%)$ & 0,2 \\
\hline Longueur moyenne de colon restant (\%) & $90 \%( \pm 10 \%)$ & $90 \%( \pm 20 \%)$ & 0,5 \\
\hline Sepsis & $9(45,0 \%)$ & $23(49,9 \%)$ & 0,8 \\
\hline Dont sepsis d'origine hépatobiliaire & $4(20,0 \%)$ & $3(6,1 \%)$ & 0,08 \\
\hline Pathologie hépatobiliaire & $16(80,0 \%)$ & $11(22,4 \%)$ & $\leq 0,001 \ddagger$ \\
\hline Dont pathologie lithiasique & $3(15,0 \%)$ & $4(8,2 \%)$ & 0,3 \\
\hline Traitements hépatotoxiques & $6(30 \%)$ & 24 (49 \%) & - \\
\hline Antibiothérapie & $6(30,0 \%)$ & $16(32,7 \%)$ & 0,1 \\
\hline Chimiothérapie & $0(0,0 \%)$ & $8(16,3 \%)$ & 0,9 \\
\hline
\end{tabular}

$\dagger$ : analyse effectuée sur $\mathrm{n}=65$ patients (données anthropométriques manquantes)

$\ddagger$ : en analyse multivariée, $\mathrm{OR}=18,9 ; \mathrm{I} \mathrm{C}_{95 \%}[3,5-100,5] ; \mathrm{p}<0,001$ 


\section{Critère cholestase 3}

La prévalence des $A B H$ avant introduction de la NP selon le critère cholestase 3 était de 29,9 $\%$. Les patients qui perturbaient leur bilan hépatique avaient significativement plus de pathologies hépatobiliaires $(80,0 \%$ vs. $22,4 \%, p<0,001)$ et ce résultat était significatif en analyse multivariée (OR $=18,9$, IC $95 \% 3,5-100,5, p<0,001)$. On notait également une proportion plus importante d'antécédent de cholécystectomie chez les patients avec ABH (100,0\% vs. $83,7 \%, p=0,05)$ mais ce résultat n'était pas retrouvé en analyse multivariée ( $\underline{\text { Tableau } 5}$ ). 
Tableau 6-Comparaison des patients avec et sans anomalies du bilan hépatique selon le critère stéatose, avant introduction de la nutrition parentérale

\begin{tabular}{|c|c|c|c|}
\hline & $\begin{array}{c}\text { Oui } \\
N=7(10,1 \%)\end{array}$ & $\begin{array}{c}\text { Non } \\
N=62(89,1 \%)\end{array}$ & $\mathbf{p}$ \\
\hline Age (années) & $55( \pm 11,4)$ & $61,9( \pm 15,0)$ & 0,2 \\
\hline $\begin{array}{ll}\text { Sexe } & \\
\text { Homme } \\
\text { Femme }\end{array}$ & $\begin{array}{c}7(100,0 \%) \\
0(0,0 \%) \\
\end{array}$ & $\begin{array}{l}37(59,7 \%) \\
25(40,3 \%)\end{array}$ & 0,03 \\
\hline $\begin{array}{l}\text { IMC moyen }\left(\mathbf{k g} / \mathrm{m}^{2}\right) \dagger \\
\text { Dénutrition sévère selon IMC } \\
\text { Dénutrition modérée selon IMC } \\
\text { Normal } \\
\text { Surpoids } \\
\text { Obésité }\end{array}$ & $\begin{array}{c}25,0( \pm 4,0) \\
0(0,0 \%) \\
0(0,0 \%) \\
3(50,0 \%) \\
3(50,0 \%) \\
(0,0 \%) \\
\end{array}$ & $\begin{array}{c}21,5( \pm 5,2) \\
16(27,1 \%) \\
8(13,6 \%) \\
24(40,7 \%) \\
7(11,9 \%) \\
4(6,8 \%) \\
\end{array}$ & $\begin{array}{c}0,1 \\
0,1 \\
0,3 \\
0,2 \\
0,05 \\
0,4 \\
\end{array}$ \\
\hline $\begin{array}{l}\text { Dénutrition selon la HAS † } \\
\text { Modérée } \\
\text { Sévère }\end{array}$ & $\begin{array}{c}0(0,0 \%) \\
1(16,7 \%)\end{array}$ & $\begin{array}{c}6(10,2 \%) \\
20(33,9 \%)\end{array}$ & $\begin{array}{l}0,3 \\
0,3\end{array}$ \\
\hline $\begin{array}{l}\text { Pathologie principale } \\
\text { Cancer } \\
\text { Pathologie hépato-bilio-pancréatique bénigne } \\
\text { Autre pathologie digestive } \\
\text { Autre }\end{array}$ & $\begin{array}{l}2(28,6 \%) \\
2(28,6 \%) \\
1(14,3 \%) \\
2(28,6 \%)\end{array}$ & $\begin{array}{c}22(35,5 \%) \\
14(22,6 \%) \\
22(35,5 \%) \\
4(6,5 \%)\end{array}$ & $\begin{array}{l}- \\
0,5 \\
0,9 \\
0,2\end{array}$ \\
\hline $\begin{array}{l}\text { Antécédent de chirurgie du tube digestif } \\
\text { Résection iléale } \\
\text { Résection colique } \\
\text { Résection iléo-colique } \\
\text { Chirurgie sus mésocolique }\end{array}$ & $\begin{array}{c}1(14,3 \%) \\
0(0,0 \%) \\
0(0,0 \%) \\
1(14,3 \%) \\
0(0,0 \%)\end{array}$ & $\begin{array}{r}16(25,8 \%) \\
5(8,2 \%) \\
4(6,5 \%) \\
2(3,2 \%) \\
5(8,1 \%)\end{array}$ & $\begin{array}{l}0,9 \\
0,9 \\
0,9 \\
0,9\end{array}$ \\
\hline Antécédent de cholécystectomie & $0(0,0 \%)$ & $8(12,9 \%)$ & 0,3 \\
\hline $\begin{array}{l}\text { Insuffisance intestinale } \\
\text { Grêle court } \\
\text { Fistule intestinale } \\
\text { Trouble moteur } \\
\text { Obstruction } \\
\text { Atteinte muqueuse }\end{array}$ & $\begin{array}{l}2(28,6 \%) \\
0(0,0 \%) \\
0(0,0 \%) \\
2(28,6 \%) \\
0(0,0 \%) \\
0(0,0 \%)\end{array}$ & $\begin{array}{c}31(50,0 \%) \\
8(12,9 \%) \\
3(4,8 \%) \\
8(12,9 \%) \\
11(17,7 \%) \\
1(1,6 \%)\end{array}$ & $\begin{array}{l}0,2 \\
0,9 \\
0,9 \\
0,9 \\
0,5 \\
0,9\end{array}$ \\
\hline NP exclusive & $1(14,3 \%)$ & $16(25,8 \%)$ & 0,5 \\
\hline Longueur moyenne de colon restant (\%) & $90 \%( \pm 10 \%)$ & $90 \%( \pm 10 \%)$ & 0,7 \\
\hline $\begin{array}{l}\text { Sepsis } \\
\text { Dont sepsis d'origine hépatobiliaire }\end{array}$ & $\begin{array}{l}4(57,1 \%) \\
1(14,3 \%)\end{array}$ & $\begin{array}{c}28(45,2 \%) \\
6(9,7 \%)\end{array}$ & $\begin{array}{l}0,5 \\
0,7\end{array}$ \\
\hline $\begin{array}{l}\text { Pathologie hépatobiliaire } \\
\text { Dont pathologie lithiasique }\end{array}$ & $\begin{array}{c}7(100,0 \%) \\
0(0,0 \%)\end{array}$ & $\begin{array}{l}20(32,3 \%) \\
7(11,3 \%)\end{array}$ & $\begin{array}{c}0,001 \\
0,3\end{array}$ \\
\hline $\begin{array}{c}\text { Traitements hépatotoxiques } \\
\text { Antibiothérapie } \\
\text { Chimiothérapie }\end{array}$ & $\begin{array}{c}2(28,6 \%) \\
2(28,6 \%) \\
0(0,0 \%)\end{array}$ & $\begin{array}{l}28(45,2 \%) \\
20(32,3 \%) \\
8(12,9 \%)\end{array}$ & $\begin{array}{l}0,5 \\
0,9\end{array}$ \\
\hline
\end{tabular}

$\dagger$ : analyse effectuée sur $n=65$ patients (données anthropométriques manquantes) 


\section{Critère stéatose}

La prévalence des $\mathrm{ABH}$ avant introduction de la NP selon le critère stéatose était de 10,1 \%. Les patients qui perturbaient leur bilan hépatique avaient significativement plus de pathologies hépato-biliaires $(100,0 \%$ vs. $32,3 \%, p<0,001)$, étaient majoritairement des hommes $(100,0 \%$ vs. 59,7 $\%, p=0,03)$ et des patients en surpoids $(42,9 \%$ vs. $11,3 \%, p=0,05)$ mais ces résultats n'étaient pas significatifs en analyse multivariée ( $\underline{\text { Tableau } 6}$ ). 
Tableau 7 - Comparaison des patients avec et sans anomalies du bilan hépatique selon le critère mixte avant introduction de la nutrition parentérale

\begin{tabular}{|c|c|c|c|}
\hline & $\begin{array}{c}\text { Oui } \\
n=33(47,1 \%)\end{array}$ & $\begin{array}{c}\text { Non } \\
n=37(52,9 \%)\end{array}$ & $\mathbf{p}$ \\
\hline Age (années) & $62,2( \pm 13,2)$ & $60,3( \pm 15,9)$ & 0,5 \\
\hline $\begin{array}{ll}\text { Sexe } & \\
\text { Homme } \\
\text { Femme }\end{array}$ & $\begin{array}{l}22(66,7 \%) \\
11(33,3 \%)\end{array}$ & $\begin{array}{l}23(62,2 \%) \\
14(37,8 \%)\end{array}$ & 0,6 \\
\hline $\begin{array}{l}\mathrm{IMC} \text { moyen }\left(\mathrm{kg} / \mathrm{m}^{2}\right)^{\dagger} \\
\text { Dénutrition sévère selon IMC } \\
\text { Dénutrition modérée selon IMC } \\
\text { Normal } \\
\text { Surpoids } \\
\text { Obésité }\end{array}$ & $\begin{array}{l}21,2( \pm 3,9) \\
7(22,6 \%) \\
4(12,9 \%) \\
15(48,4 \%) \\
5(15,2 \%) \\
0(16,1 \%)\end{array}$ & $\begin{array}{l}22,2( \pm 6,1) \\
9(25,7 \%) \\
5(14,2 \%) \\
12(34,3 \%) \\
5(14,3 \%) \\
4(11,4 \%)\end{array}$ & $\begin{array}{l}0,4 \\
0,8 \\
0,7 \\
0,7 \\
0,8 \\
0,1\end{array}$ \\
\hline $\begin{array}{l}\text { Dénutrition selon la HAS † } \\
\text { Modérée } \\
\text { Sévère }\end{array}$ & $\begin{array}{l}1(3,1 \%) \\
9(29,0 \%)\end{array}$ & $\begin{array}{c}5(14,2 \%) \\
12(34,3 \%)\end{array}$ & $\begin{array}{l}0,1 \\
0,1\end{array}$ \\
\hline $\begin{array}{l}\text { Pathologie principale } \\
\text { Cancer } \\
\text { Pathologie hépato-bilio-pancréatique bénigne } \\
\text { Autre pathologie digestive } \\
\text { Autre }\end{array}$ & $\begin{array}{c}13(39,4 \%) \\
10(30,3 \%) \\
7(21,2 \%) \\
3(9,1 \%)\end{array}$ & $\begin{array}{c}12(32,4 \%) \\
6(16,2 \%) \\
16(43,2 \%) \\
3(8,1 \%)\end{array}$ & $\begin{array}{c}- \\
0,7 \\
0,2 \\
0,07\end{array}$ \\
\hline $\begin{array}{l}\text { Antécédent de chirurgie du tube digestif } \\
\text { Résection iléale } \\
\text { Résection colique } \\
\text { Résection iléo-colique } \\
\text { Chirurgie sus mésocolique }\end{array}$ & $\begin{array}{l}6(18,2 \%) \\
1(3,0 \%) \\
1(3,0 \%) \\
2(6,1 \%) \\
2(6,1 \%)\end{array}$ & $\begin{array}{c}12(32,4 \%) \\
4(10,8 \%) \\
4(10,8 \%) \\
1(2,7 \%) \\
3(8,1 \%)\end{array}$ & $\begin{array}{l}0,3 \\
0,2 \\
0,2 \\
0,3\end{array}$ \\
\hline Antécédent de cholécystectomie & $3(9,1 \%)$ & $5(13,5 \%)$ & 0,5 \\
\hline $\begin{array}{l}\text { Insuffisance intestinale } \\
\text { Grêle court } \\
\text { Fistule intestinale } \\
\text { Trouble moteur } \\
\text { Obstruction } \\
\text { Atteinte muqueuse }\end{array}$ & $\begin{array}{c}11(33,3 \%) \\
2(6,1 \%) \\
0(0,0 \%) \\
5(15,2 \%) \\
4(12,1 \%) \\
0(0,0 \%)\end{array}$ & $\begin{array}{c}23(62,2 \%) \\
6(16,2 \%) \\
3(8,1 \%) \\
5(13,5 \%) \\
8(21,6 \%) \\
1(2,7 \%)\end{array}$ & $\begin{array}{c}0,01 \\
0,2 \\
0,9 \\
0,9 \\
0,9 \\
0,1\end{array}$ \\
\hline NP exclusive & $5(15,2 \%)$ & $13(35,1 \%)$ & 0,05 \\
\hline Longueur moyenne de colon restant (\%) & $90 \%( \pm 10 \%)$ & $90 \%( \pm 20 \%)$ & 0,4 \\
\hline $\begin{array}{l}\text { Sepsis } \\
\text { Dont sepsis d'origine hépatobiliaire }\end{array}$ & $\begin{array}{c}16(48,5 \%) \\
7(21,2 \%)\end{array}$ & $\begin{array}{c}16(43,2 \%) \\
0(0,0 \%)\end{array}$ & $\begin{array}{c}0,6 \\
0,003\end{array}$ \\
\hline $\begin{array}{l}\text { Pathologie hépatobiliaire } \\
\text { Dont pathologie lithiasique }\end{array}$ & $\begin{array}{l}23(69,7 \%) \\
6(18,2 \%)\end{array}$ & $\begin{array}{c}4(10,8 \%) \\
1(2,7 \%)\end{array}$ & $\frac{<0,001 \ddagger}{0,03}$ \\
\hline $\begin{array}{c}\text { Traitements hépatotoxiques } \\
\text { Antibiothérapie } \\
\text { Chimiothérapie }\end{array}$ & $\begin{array}{c}18(54,5 \%) \\
12(36,4 \%) \\
6(18,2 \%)\end{array}$ & $\begin{array}{c}13(35,1 \%) \\
10(24,0 \%) \\
3(8,1 \%)\end{array}$ & $\begin{array}{c}0,08 \\
0,9\end{array}$ \\
\hline
\end{tabular}

$\dagger$ : analyse effectuée sur $\mathrm{n}=65$ patients (données anthropométriques manquantes)

$\ddagger$ : en analyse multivariée, $O R=10,1 ; I C_{95 \%}[2,4-48,5] ; p<0,001$ 


\section{Critère mixte}

La prévalence des $\mathrm{ABH}$ avant introduction de la NP selon le critère mixte était de $47,1 \%$. Les patients qui perturbaient leur bilan hépatique avaient significativement plus de pathologies hépatobiliaires $(69,7 \%$ vs. $10,8 \%, p<0,001)$ et ce résultat était significatif en analyse multivariée (OR $=10,1$, IC $95 \%$ 2,4-48,5, p < 0,001). On notait également une proportion moins importante d'insuffisance intestinale ( $33,3 \%$ vs. $62,2 \%, p=0,01)$, moins de patients redevables d'une NP exclusive $(15,2 \%$ vs. $62,2 \%)$, davantage de sepsis d'origine hépato-biliaire $(21,2 \%$ vs. $0,0 \%, p=0,003)$ et davantage de pathologies lithiasiques $(18,2 \%$ vs. $17,7 \%, p=0,03)$ mais ces résultats n'étaient pas retrouvés en analyse multivariée ( $\underline{\text { Tableau } 7)}$. 


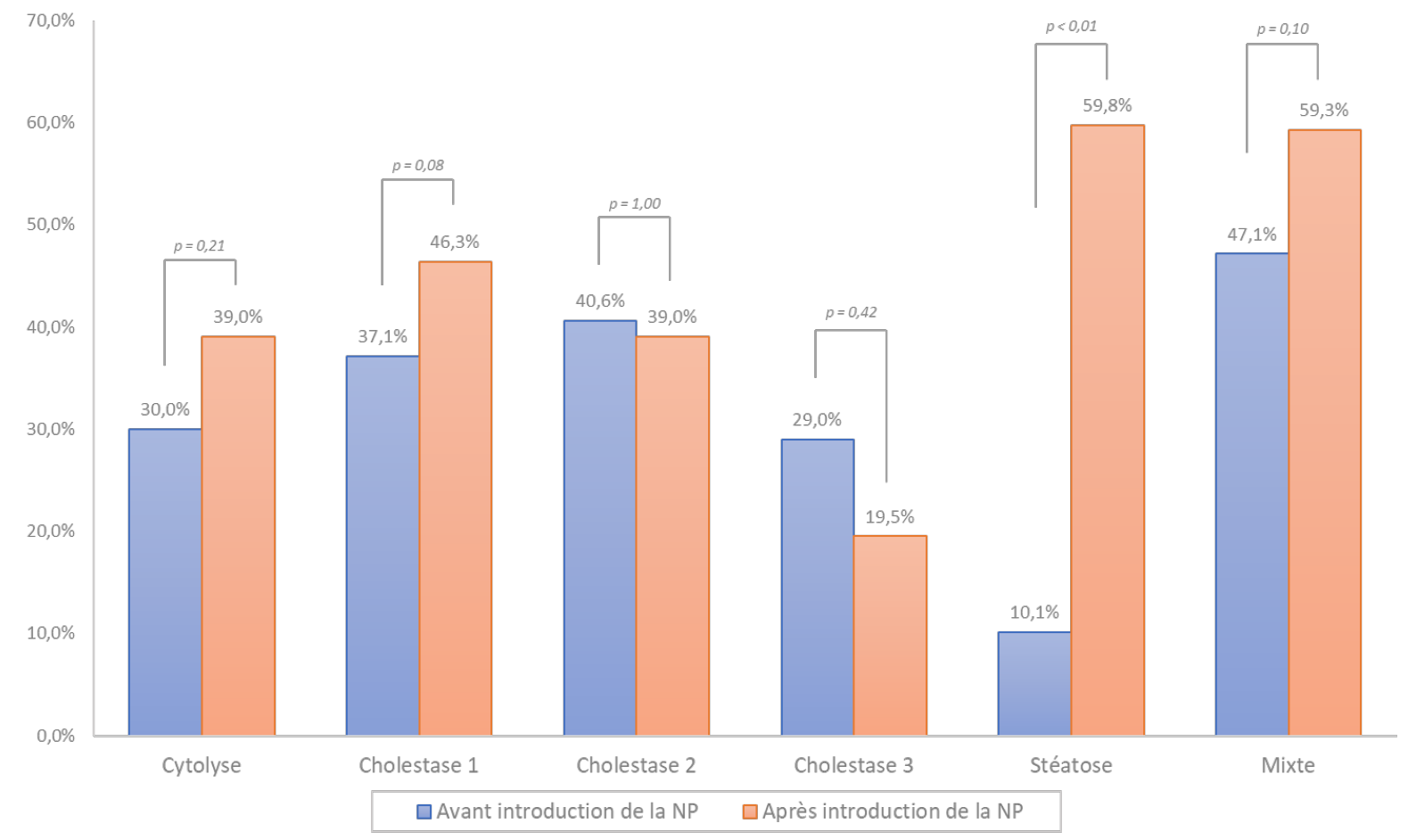

Figure 3-Comparaison de la prévalence des anomalies du bilan hépatique avant et après introduction de la nutrition parentérale 


\section{Évolution de la prévalence}

La prévalence médiane des ABH avant la NP était de 30,4\% (IQ = 29,5-38,1\%). La prévalence médiane des $A B H$ après introduction de la NP était de $42,7 \%(I Q=39,0-56,1 \%)$. La prévalence des $A B H$ selon le critère stéatose était significativement plus importante après introduction de la NP $(10,1$ $\%$ vs. $59,8 \%, p<0,01)$. La prévalence selon les autres critères biologiques ne variait pas de manière significative après introduction de la NP. On remarquait cependant une tendance à la majoration des $\mathrm{ABH}$ selon le critère cytolyse, le critère cholestase 1 et le critère mixte (Figure $\mathbf{3}$ ). 


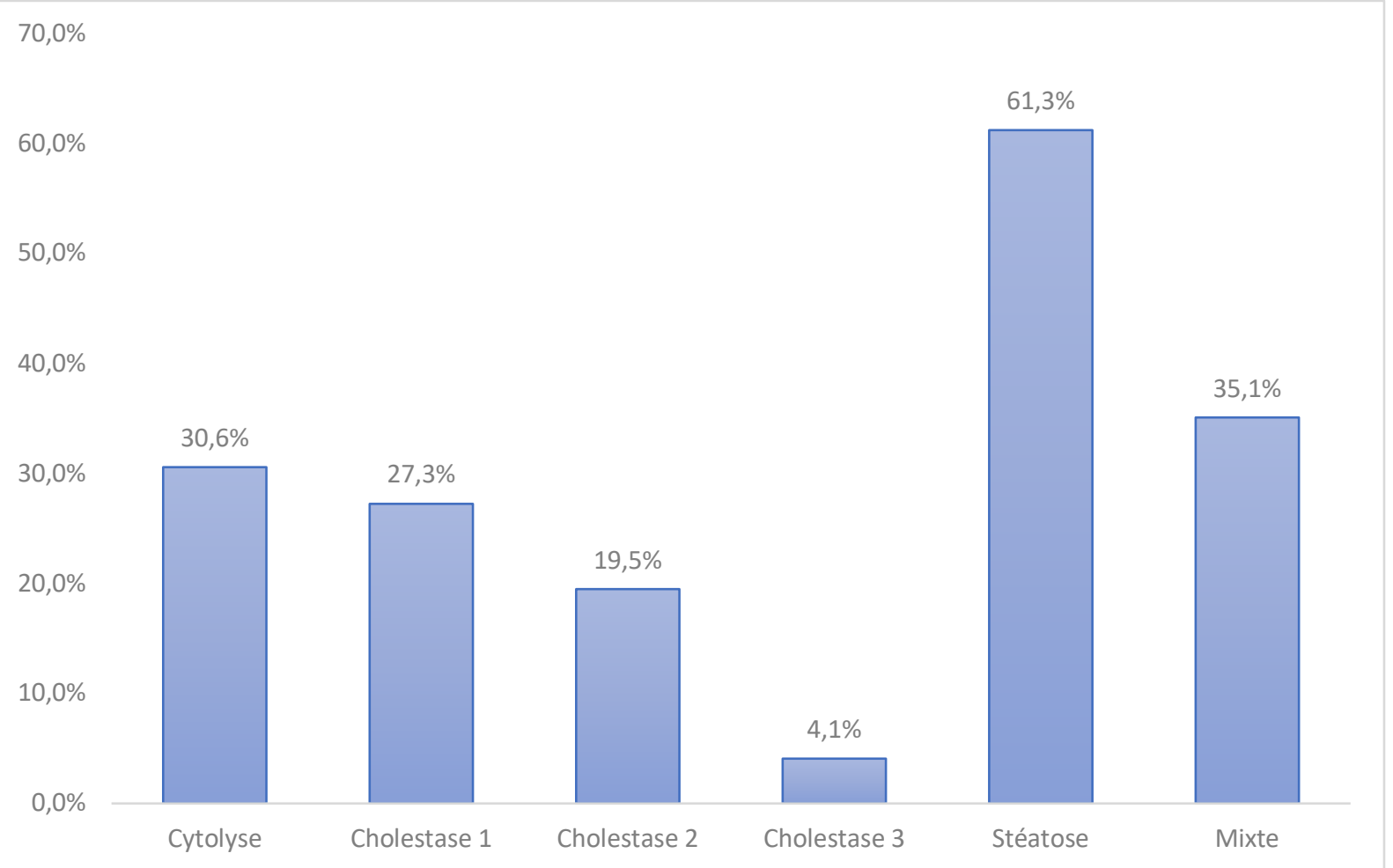

Fiqure 4-Incidence des $A B H$ selon chaque critère biologique 
Nous avons calculé l'incidence d'anomalies du bilan hépatiques selon chaque critère biologique (Figure 4) . On remarque une grande variabilité de l'incidence selon le critère biologique ( $4,1 \%$ à $61,3 \%)$ avec une médiane à $28,9 \%$ (IQ = 23,4-34,0\%). 
Tableau 8-Comparaison des patients avec et sans anomalies du bilan hépatique incidentes selon le critère cytolyse après introduction de la nutrition parentérale

\begin{tabular}{|c|c|c|c|}
\hline & $\begin{array}{c}\text { Oui } \\
n=15(30,6 \%)\end{array}$ & $\begin{array}{c}\text { Non } \\
\mathrm{n}=34(60,4 \%)\end{array}$ & $\mathbf{p}$ \\
\hline Age (années) & $60( \pm 14,2)$ & $63( \pm 15,8)$ & 0,5 \\
\hline \multicolumn{4}{|l|}{ Sexe } \\
\hline Homme & $8(53,3 \%)$ & $22(64,7 \%)$ & \\
\hline Femme & $7(46,7 \%)$ & $12(35,3 \%)$ & 0,4 \\
\hline IMC moyen $\left(\mathrm{kg} / \mathrm{m}^{2}\right)^{\dagger}$ & $21,9( \pm 5,8)$ & $22,1( \pm 5,3)$ & 0,9 \\
\hline Dénutrition sévère selon IMC & $3(21,4 \%)$ & $7(21,9 \%)$ & 0,9 \\
\hline Dénutrition modérée selon IMC & $1(7,1 \%)$ & $6(18,8 \%)$ & - \\
\hline Normal & $8(57,1 \%)$ & $11(34,4 \%)$ & 0,4 \\
\hline Surpoids & $1(7,1 \%)$ & $5(15,6 \%)$ & - \\
\hline Obésité & $1(7,1 \%)$ & $3(9,4 \%)$ & - \\
\hline \multicolumn{4}{|l|}{ Dénutrition selon la HAS † } \\
\hline Modérée & $2(14,2 \%)$ & $4(12,5 \%)$ & 0,8 \\
\hline Sévère & $5(35,7 \%)$ & $12(37,5 \%)$ & 0,8 \\
\hline \multicolumn{4}{|l|}{ Pathologie principale } \\
\hline Cancer & $10(66,7 \%)$ & $10(29,4 \%)$ & - \\
\hline Pathologie hépato-bilio-pancréatique bénigne & $2(13,3 \%)$ & $7(20,6 \%)$ & 0,9 \\
\hline Autre pathologie digestive & $3(20,0 \%)$ & $15(44,1 \%)$ & 0,6 \\
\hline Autre & $0(0,0 \%)$ & $2(5,9 \%)$ & 0,4 \\
\hline Antécédent de chirurgie du tube digestif & $3(20 \%)$ & $12(35,3 \%)$ & \\
\hline Résection iléale & $0(0,0 \%)$ & $4(11,8 \%)$ & 0,9 \\
\hline Résection colique & $1(6,7 \%)$ & $3(8,8 \%)$ & 0,9 \\
\hline Résection iléo-colique & $0(0,0 \%)$ & $2(5,9 \%)$ & 0,9 \\
\hline Chirurgie sus mésocolique & $2(13,3 \%)$ & $3(8,8 \%)$ & 0,9 \\
\hline Antécédent de cholécystectomie & $2(13,3 \%)$ & $5(14,7 \%)$ & 0,8 \\
\hline Insuffisance intestinale & $10(66,7 \%)$ & $12(35,3 \%)$ & 0,04 \\
\hline Grêle court & $0(0,0 \%)$ & $7(20,6 \%)$ & 0,9 \\
\hline Fistule intestinale & $1(6,7 \%)$ & $2(5,9 \%)$ & 0,9 \\
\hline Trouble moteur & $1(6,7 \%)$ & $5(14,7 \%)$ & 0,9 \\
\hline Obstruction & $3(20,0 \%)$ & $7(20,6 \%)$ & 0,9 \\
\hline Atteinte muqueuse & $0(0,0 \%)$ & $1(2,9 \%)$ & 0,9 \\
\hline NP exclusive & $6(40,0 \%)$ & $8(23,5 \%)$ & 0,2 \\
\hline Longueur moyenne de colon restant (\%) & $90 \%( \pm 7 \%)$ & $80 \%( \pm 20 \%)$ & 0,2 \\
\hline Sepsis & $7(46,7 \%)$ & $15(44,1 \%)$ & 0,8 \\
\hline Dont sepsis d'origine hépatobiliaire & $1(6,7 \%)$ & $3(8,8 \%)$ & 0,7 \\
\hline Pathologie hépatobiliaire & $6(40,0 \%)$ & $6(17,6 \%)$ & 0,09 \\
\hline Dont pathologie lithiasique & $0(0,0 \%)$ & $4(11,8 \%)$ & - \\
\hline Traitements hépatotoxiques & $8(53,3 \%)$ & $1544,1 \%)$ & \\
\hline Antibiothérapie & $4(26,7 \%)$ & $11(32,4 \%)$ & 0,4 \\
\hline Chimiothérapie & $4(26,7 \%)$ & $4(11,8 \%)$ & 0,4 \\
\hline Apports caloriques parentéraux moyens ( $\mathrm{kcal} / \mathrm{kg} / \mathrm{j})$ & $25,9( \pm 8,8)$ & $30,6( \pm 12,5)$ & 0,2 \\
\hline Dont glucides $(\mathrm{g} / \mathrm{kg} / \mathrm{j})$ & $3,1( \pm 1,0)$ & $3,7( \pm 1,5)$ & 0,1 \\
\hline Dont protides $(\mathrm{g} / \mathrm{kg} / \mathrm{j})$ & $1,0( \pm 0,3$ & $1,4( \pm 0,4)$ & 0,2 \\
\hline Dont lipides $(\mathrm{g} / \mathrm{kg} / \mathrm{j})$ & $0,9( \pm 0,3)$ & $1,0( \pm 0,4)$ & 0,2 \\
\hline
\end{tabular}

+ : analyse effectuée sur $n=46$ patients (données anthropométriques manquantes) 


\section{Facteurs associés aux anomalies du bilan hépatiques incidentes}

\section{Critère cytolyse}

L'incidence de survenue des $\mathrm{ABH}$ selon le critère cytolyse était de $30,6 \%$. Ces patients avaient significativement plus de pathologies hépato-biliaires ( $71,4 \%$ vs. $24,5 \%, p<0,001)$ et significativement plus de patients présentant une insuffisance intestinale $(66,7 \%$ vs. $35,3 \%, p=0,04)$ mais cela n'était pas retrouvé en analyse multivariée. II n'y avait par ailleurs pas d'autre différence significative entre les cas incidents et les non-incidents ( $\underline{\text { Tableau } 8}$ ). 
Tableau 9-Comparaison des patients avec et sans anomalies du bilan hépatique incidentes selon le critère cholestase 1 après introduction de la nutrition parentérale

\begin{tabular}{|c|c|c|c|}
\hline & $\begin{array}{c}\text { Oui } \\
n=12(27,3 \%)\end{array}$ & $\begin{array}{c}\text { Non } \\
n=32(72,7 \%)\end{array}$ & $\mathbf{p}$ \\
\hline Age (années) & $63( \pm 12,3)$ & $60( \pm 16,8)$ & 0,4 \\
\hline $\begin{array}{ll}\text { Sexe } & \\
\text { Homme } \\
\text { Femme }\end{array}$ & $\begin{array}{l}9(75 \%) \\
3(25 \%)\end{array}$ & $\begin{array}{c}2062,5 \%) \\
12(37,5 \%)\end{array}$ & 0,4 \\
\hline $\begin{array}{l}\text { IMC moyen }\left(\mathbf{k g} / \mathrm{m}^{2}\right) \dagger \\
\text { Dénutrition sévère selon IMC } \\
\text { Dénutrition modérée selon IMC } \\
\text { Normal } \\
\text { Surpoids } \\
\text { Obésité }\end{array}$ & $\begin{array}{c}18,3( \pm 4,5) \\
7(58,3 \%) \\
1(8,3 \%) \\
3(25,0 \%) \\
1(8,3 \%) \\
0(0 \%) \\
\end{array}$ & $\begin{array}{c}23,2( \pm 6,1) \\
6(20,0 \%) \\
4(13,3 \%) \\
11(36,7 \%) \\
5(16,6 \%) \\
4(13,3 \%) \\
\end{array}$ & $\begin{array}{c}0,03 \\
0,01 \\
0,7 \\
0,09 \\
0,5 \\
0,9\end{array}$ \\
\hline $\begin{array}{l}\text { Dénutrition selon la HAS † } \\
\text { Modérée } \\
\text { Sévère }\end{array}$ & $\begin{array}{c}0(0 \%) \\
5(41,7 \%)\end{array}$ & $\begin{array}{c}5(16,6 \%) \\
11(36,7 \%)\end{array}$ & - \\
\hline $\begin{array}{l}\text { Pathologie principale } \\
\text { Cancer } \\
\text { Pathologie hépato-bilio-pancréatique bénigne } \\
\text { Autre pathologie digestive } \\
\text { Autre }\end{array}$ & $\begin{array}{c}4(33,3 \%) \\
3(25 \%) \\
4(33,3 \%) \\
1(8,3 \%)\end{array}$ & $\begin{array}{c}10(31,3 \%) \\
5(15,6 \%) \\
13(40,6 \%) \\
4(12,5 \%)\end{array}$ & $\begin{array}{l}- \\
0,8 \\
0,4 \\
0,7\end{array}$ \\
\hline $\begin{array}{l}\text { Antécédent de chirurgie du tube digestif } \\
\text { Résection iléale } \\
\text { Résection colique } \\
\text { Résection iléo-colique } \\
\text { Chirurgie sus mésocolique }\end{array}$ & $\begin{array}{l}4(33,3 \%) \\
0(0 \%) \\
3(25 \%) \\
1(8,3 \%) \\
0(0 \%)\end{array}$ & $\begin{array}{l}9(28,1 \%) \\
4(12,5 \%) \\
1(3,1 \%) \\
1(3,1 \%) \\
3(9,4 \%)\end{array}$ & $\begin{array}{l}0,9 \\
0,9 \\
0,9 \\
0,9\end{array}$ \\
\hline Antécédent de cholécystectomie & $0(0 \%)$ & $5(15,6 \%)$ & 0,9 \\
\hline $\begin{array}{l}\text { Insuffisance intestinale } \\
\text { Grêle court } \\
\text { Fistule intestinale } \\
\text { Trouble moteur } \\
\text { Obstruction } \\
\text { Atteinte muqueuse }\end{array}$ & $\begin{array}{c}4(33,3 \%) \\
2(16,7 \%) \\
1(8,3 \%) \\
3(25 \%) \\
2(16,7 \%) \\
0(0 \%)\end{array}$ & $\begin{array}{c}16(50 \%) \\
4(12,5 \%) \\
2(6,3 \%) \\
3(9,4 \%) \\
6(18,8 \%) \\
1(3,1 \%)\end{array}$ & $\begin{array}{c}- \\
0,9 \\
0,9 \\
0,9 \\
0,9 \\
0,9\end{array}$ \\
\hline NP exclusive & $3(25 \%)$ & $10(31,3 \%)$ & 0,6 \\
\hline Longueur moyenne de colon restant (\%) & $80 \%( \pm 20 \%)$ & $90 \%( \pm 10 \%)$ & 0,2 \\
\hline $\begin{array}{l}\text { Sepsis } \\
\quad \text { Dont sepsis d'origine hépatobiliaire }\end{array}$ & $\begin{array}{c}5(41,7 \%) \\
0(0 \%)\end{array}$ & $\begin{array}{c}14(43,8 \%) \\
0(0 \%)\end{array}$ & 0,9 \\
\hline $\begin{array}{l}\text { Pathologie hépatobiliaire } \\
\text { Dont pathologie lithiasique }\end{array}$ & $\begin{array}{l}4(33,3 \%) \\
1(8,3 \%)\end{array}$ & $\begin{array}{l}(12,5 \%) \\
2(6,3 \%)\end{array}$ & $\begin{array}{l}0,1 \\
0,8\end{array}$ \\
\hline $\begin{array}{l}\text { Traitements hépatotoxiques } \\
\text { Antibiothérapie } \\
\text { Chimiothérapie }\end{array}$ & $\begin{array}{c}6(50 \%) \\
4(33,3 \%) \\
2(16,7 \%)\end{array}$ & $\begin{array}{c}10(31,3 \%) \\
8(25 \%) \\
2(6,3 \%)\end{array}$ & $\begin{array}{l}0,2 \\
0,9\end{array}$ \\
\hline $\begin{array}{l}\text { Apports caloriques parentéraux moyens }(\mathrm{kcal} / \mathrm{kg} / \mathrm{j}) \\
\text { Dont glucides }(\mathrm{g} / \mathrm{kg} / \mathrm{j}) \\
\text { Dont protides }(\mathrm{g} / \mathrm{kg} / \mathrm{j}) \\
\text { Dont lipides }(\mathrm{g} / \mathrm{kg} / \mathrm{j})\end{array}$ & $\begin{array}{r}34,5( \pm 18,2) \\
4,2( \pm 2,2) \\
1,3( \pm 0,7) \\
1,2( \pm 0,6)\end{array}$ & $\begin{array}{l}28,8( \pm 7,4) \\
3,5( \pm 0,9) \\
1,1( \pm 0,2) \\
1,0( \pm 0,2)\end{array}$ & $\begin{array}{l}0,1 \\
0,1 \\
0,1 \\
0,1\end{array}$ \\
\hline
\end{tabular}

+ : analyse effectuée sur $n=42$ patients (données anthropométriques manquantes) 


\section{Critère cholestase 1}

L'incidence de survenue des ABH selon le critère cholestase 1 était de $27,3 \%$. Les patients avec $A B H$ après introduction de la NP avaient un IMC significativement plus bas que les patients sans $A B H$ $\left(18,3 \pm 4,5 \mathrm{~kg} / \mathrm{m}^{2}\right.$ vs. $\left.23,2 \pm 6,1 \mathrm{~kg} / \mathrm{m}^{2}\right)$ et avaient également une proportion plus importante de patients avec un IMC répondant à la définition HAS de la dénutrition sévère $(58,3 \%$ vs. $20,0 \%)$. Les patients avec $A B H$ présentaient également une NP depuis plus longtemps que les patients sans $A B H$ mais cela n'était pas significatif $(23,6 \pm 12,2$ j vs. $16,5 \pm 10,8, p=0,08)$. ( $\underline{\text { Tableau } 9 \text { ). }}$. 
Tableau 10-Comparaison des patients avec et sans anomalies du bilan hépatique incidentes selon le critère cholestase 2 après introduction de la nutrition parentérale

\begin{tabular}{|c|c|c|c|}
\hline & $\begin{array}{c}\text { Oui } \\
\mathrm{n}=8(19.5 \%)\end{array}$ & $\begin{array}{c}\text { Non } \\
n=33(80.5 \%)\end{array}$ & $\mathbf{p}$ \\
\hline Age (années) & $60( \pm 15)$ & $61( \pm 15)$ & \\
\hline $\begin{array}{ll}\text { Sexe } & \\
& \text { Homme } \\
& \text { Femme }\end{array}$ & $\begin{array}{l}4(50,0 \%) \\
4(50,0 \%)\end{array}$ & $\begin{array}{l}19(57,6 \%) \\
14(42,4 \%)\end{array}$ & 0,6 \\
\hline $\begin{array}{l}\text { IMC moyen }\left(\mathrm{kg} / \mathrm{m}^{2}\right) \dagger \\
\text { Dénutrition sévère selon IMC } \\
\text { Dénutrition modérée selon IMC } \\
\text { Normal } \\
\text { Surpoids } \\
\text { Obésité }\end{array}$ & $\begin{array}{l}21,4( \pm 8,8) \\
4(50,0 \%) \\
0(0 \%) \\
2(50,0 \%) \\
1(12,5 \%) \\
1(87,5 \%)\end{array}$ & $\begin{array}{c}22,2( \pm 5,0) \\
5(16,1 \%) \\
3(9,7 \%) \\
16(51,6 \%) \\
4(12,9 \%) \\
3(9,7 \%)\end{array}$ & $\begin{array}{l}0,7 \\
0,04 \\
0,9 \\
0,2 \\
0,9 \\
0,7\end{array}$ \\
\hline $\begin{array}{l}\text { Dénutrition selon la HAS † } \\
\text { Modérée } \\
\text { Sévère }\end{array}$ & $\begin{array}{c}0(0 \%) \\
1(12,5 \%)\end{array}$ & $\begin{array}{l}3(9,7 \%) \\
9(29,0 \%)\end{array}$ & $\begin{array}{l}0,9 \\
0,3\end{array}$ \\
\hline $\begin{array}{l}\text { Pathologie principale } \\
\text { Cancer } \\
\text { Pathologie hépato-bilio-pancréatique bénigne } \\
\text { Autre pathologie digestive } \\
\text { Autre }\end{array}$ & $\begin{array}{c}5(62,5 \%) \\
0(0 \%) \\
1(12,5 \%) \\
2(25 \%) \\
\end{array}$ & $\begin{array}{c}12(36,4 \%) \\
5(15,2 \%) \\
15(45,5 \%) \\
1(3 \%)\end{array}$ & $\begin{array}{l}- \\
0,9 \\
0,9 \\
0,9\end{array}$ \\
\hline $\begin{array}{l}\text { Antécédent de chirurgie du tube digestif } \\
\text { Résection iléale } \\
\text { Résection colique } \\
\text { Résection iléo-colique } \\
\text { Chirurgie sus mésocolique }\end{array}$ & $\begin{array}{c}4(50 \%) \\
0(0 \%) \\
2(25 \%) \\
1(12,5 \%) \\
1(12,5 \%)\end{array}$ & $\begin{array}{l}7(21,1 \%) \\
4(12,1 \%) \\
0(0 \%) \\
0(0 \%) \\
3(9,1 \%)\end{array}$ & $\begin{array}{l}- \\
0,9 \\
0,9 \\
0,9 \\
0,9\end{array}$ \\
\hline Antécédent de cholécystectomie & $1(12,5 \%)$ & $6(18,2 \%)$ & 0,7 \\
\hline $\begin{array}{l}\text { Insuffisance intestinale } \\
\text { Grêle court } \\
\text { Fistule intestinale } \\
\text { Trouble moteur } \\
\text { Obstruction } \\
\text { Atteinte muqueuse }\end{array}$ & $\begin{array}{c}6(75,0 \%) \\
2(25,0 \%) \\
0(0 \%) \\
0(0 \%) \\
0(0 \%) \\
0(0 \%)\end{array}$ & $\begin{array}{c}16(48,5 \%) \\
3(9,1 \%) \\
3(9,1 \%) \\
4(12,1 \%) \\
6(18,2 \%) \\
1(3 \%)\end{array}$ & $\begin{array}{c}- \\
0,9 \\
0,9 \\
0,9 \\
0,9 \\
0,9\end{array}$ \\
\hline NP exclusive & $0(0 \%)$ & $13(39,4 \%)$ & 0,9 \\
\hline Longueur moyenne de colon restant (\%) & $80 \%( \pm 30 \%)$ & $90 \%( \pm 10 \%)$ & 0,1 \\
\hline $\begin{array}{l}\text { Sepsis } \\
\text { Dont sepsis d'origine hépatobiliaire }\end{array}$ & $\begin{array}{c}4(50,0 \%) \\
0(0 \%)\end{array}$ & $\begin{array}{c}13(39,4 \%) \\
1(3 \%)\end{array}$ & $\begin{array}{l}0,5 \\
0,9\end{array}$ \\
\hline $\begin{array}{l}\text { Pathologie hépatobiliaire } \\
\text { Dont pathologie lithiasique }\end{array}$ & $\begin{array}{c}3(37,5 \%) \\
0(0 \%)\end{array}$ & $\begin{array}{l}5(15,2 \%) \\
2(6,1 \%)\end{array}$ & $\begin{array}{l}0,1 \\
0,9\end{array}$ \\
\hline $\begin{array}{c}\text { Traitements hépatotoxiques } \\
\text { Antibiothérapie } \\
\text { Chimiothérapie }\end{array}$ & $\begin{array}{l}4(25 \%) \\
1(12,5 \%) \\
1(12,5 \%)\end{array}$ & $\begin{array}{l}17(51,5 \%) \\
10(30,3 \%) \\
7(21,2 \%)\end{array}$ & $\begin{array}{l}0,1 \\
0,4\end{array}$ \\
\hline $\begin{array}{l}\text { Apports caloriques parentéraux moyens }(\mathrm{kcal} / \mathrm{kg} / \mathrm{j}) \\
\text { Dont glucides }(\mathrm{g} / \mathrm{kg} / \mathrm{j}) \\
\text { Dont protides }(\mathrm{g} / \mathrm{kg} / \mathrm{j}) \\
\text { Dont lipides }(\mathrm{g} / \mathrm{kg} / \mathrm{j})\end{array}$ & $\begin{array}{l}30,5( \pm 14,6) \\
3,7( \pm 1,8) \\
1,1( \pm 0,5) \\
1,0( \pm 0,5)\end{array}$ & $\begin{array}{l}29,2( \pm 8,7) \\
3,6( \pm 1,0) \\
1,3( \pm 0,3) \\
1,0( \pm 0,3)\end{array}$ & $\begin{array}{l}0,7 \\
0,7 \\
0,7 \\
0,6\end{array}$ \\
\hline
\end{tabular}

$\dagger:$ analyse effectuée sur $n=42$ patients (données anthropométriques manquantes) 
L'incidence de survenue des ABH selon le critère cholestase 2 était de 19,5\%. Les patients avec $A B H$ après introduction de la NP avaient une proportion plus importante de patients avec un IMC répondant à la définition HAS de la dénutrition sévère (50,0 \%vs. 16,1 \%) ( $\underline{\text { Tableau } 10}$ ). 
Tableau 11 -Comparaison des patients avec et sans anomalies du bilan hépatique incidentes selon le critère cholestase 3 après introduction de la nutrition parentérale

\begin{tabular}{|c|c|c|c|}
\hline & $\begin{array}{c}\text { Oui } \\
n=2(4.1 \%)\end{array}$ & $\begin{array}{c}\text { Non } \\
n=47(95.9 \%)\end{array}$ & $\mathbf{p}$ \\
\hline Age (années) & $66( \pm 9,8)$ & $61( \pm 14,7)$ & - \\
\hline $\begin{array}{l}\text { Sexe } \\
\\
\text { Homme } \\
\text { Femme }\end{array}$ & $\begin{array}{l}1(50,0 \%) \\
1(50,0 \%)\end{array}$ & $\begin{array}{l}28(59,6 \%) \\
19(40,4 \%)\end{array}$ & - \\
\hline $\begin{array}{l}\mathrm{IMC} \text { moyen }\left(\mathrm{kg} / \mathrm{m}^{2}\right) \dagger \\
\text { Dénutrition sévère selon IMC } \\
\text { Dénutrition modérée selon IMC } \\
\text { Normal } \\
\text { Surpoids } \\
\text { Obésité }\end{array}$ & $\begin{array}{c}19,4( \pm 4,7) \\
1(50,0 \%) \\
0(0,0 \%) \\
1(50,0 \%) \\
0(0,0 \%) \\
0(0,0 \%)\end{array}$ & $\begin{array}{c}21,8( \pm 5,5) \\
10(22,2 \%) \\
7(15,6 \%) \\
19(42,2 \%) \\
5(11,1 \%) \\
4(8,9 \%)\end{array}$ & $\begin{array}{l}- \\
- \\
- \\
- \\
-\end{array}$ \\
\hline $\begin{array}{l}\text { Dénutrition selon la HAS † } \\
\text { Modérée } \\
\text { Sévère }\end{array}$ & $\begin{array}{l}1(50,0 \%) \\
1(50,0 \%)\end{array}$ & $\begin{array}{c}4(8,9 \%) \\
14(31,1 \%)\end{array}$ & - \\
\hline $\begin{array}{l}\text { Pathologie principale } \\
\text { Cancer } \\
\text { Pathologie hépato-bilio-pancréatique bénigne } \\
\text { Autre pathologie digestive } \\
\text { Autre }\end{array}$ & $\begin{array}{c}1(50,0 \%) \\
0(0 \%) \\
0(0 \%) \\
1(50,0 \%)\end{array}$ & $\begin{array}{c}17(36,2 \%) \\
8(17 \%) \\
19(40,4 \%) \\
3(6,4 \%)\end{array}$ & $\begin{array}{l}- \\
- \\
-\end{array}$ \\
\hline $\begin{array}{l}\text { Antécédent de chirurgie du tube digestif } \\
\text { Résection iléale } \\
\text { Résection colique } \\
\text { Résection iléo-colique } \\
\text { Chirurgie sus mésocolique }\end{array}$ & $\begin{array}{l}0(0 \%) \\
0(0 \%) \\
0(0 \%) \\
0(0 \%) \\
0(0 \%)\end{array}$ & $\begin{array}{c}13(27,7 \%) \\
5(10,6 \%) \\
2(4,3 \%) \\
2(4,3 \%) \\
4(8,5 \%) \\
\end{array}$ & $\begin{array}{l}- \\
- \\
-\end{array}$ \\
\hline Antécédent de cholécystectomie & $0(0 \%)$ & $8(17 \%)$ & - \\
\hline $\begin{array}{l}\text { Insuffisance intestinale } \\
\text { Grêle court } \\
\text { Fistule intestinale } \\
\text { Trouble moteur } \\
\text { Obstruction } \\
\text { Atteinte muqueuse }\end{array}$ & $\begin{array}{c}1(50,0 \%) \\
0(0 \%) \\
0(0 \%) \\
0(0 \%) \\
1(50,0 \%) \\
0(0 \%)\end{array}$ & $\begin{array}{c}22(46,8 \%) \\
7(14,9 \%) \\
3(6,4 \%) \\
5(10,6 \%) \\
9(19,1 \%) \\
1(2,1 \%)\end{array}$ & $\begin{array}{l}- \\
- \\
- \\
- \\
- \\
-\end{array}$ \\
\hline NP exclusive & $1(50,0 \%)$ & $13(27,7 \%)$ & - \\
\hline Longueur moyenne de colon restant (\%) & $100,0 \%( \pm 0 \%)$ & $90 \%( \pm 20 \%)$ & - \\
\hline $\begin{array}{l}\text { Sepsis } \\
\quad \text { Dont sepsis d'origine hépatobiliaire }\end{array}$ & $\begin{array}{c}2(100,0 \%) \\
1(50,0 \%)\end{array}$ & $\begin{array}{c}21(44,7 \%) \\
2(4,3 \%)\end{array}$ & - \\
\hline $\begin{array}{l}\text { Pathologie hépatobiliaire } \\
\text { Dont pathologie lithiasique }\end{array}$ & $\begin{array}{l}1(50,0 \%) \\
0(0,0 \%)\end{array}$ & $\begin{array}{c}10(21,3 \%) \\
4(8,5 \%)\end{array}$ & - \\
\hline $\begin{array}{l}\text { Traitements hépatotoxiques } \\
\text { Antibiothérapie } \\
\text { Chimiothérapie }\end{array}$ & $\begin{array}{c}1(50 \%) \\
1(50,0 \%) \\
0(0,0 \%)\end{array}$ & $\begin{array}{c}23(48,9 \%) \\
15(31,9 \%) \\
8(17 \%)\end{array}$ & - \\
\hline $\begin{array}{l}\text { Apports caloriques parentéraux moyens }(\mathrm{kcal} / \mathrm{kg} / \mathrm{j}) \\
\text { Dont glucides }(\mathrm{g} / \mathrm{kg} / \mathrm{j}) \\
\text { Dont protides }(\mathrm{g} / \mathrm{kg} / \mathrm{j}) \\
\text { Dont lipides }(\mathrm{g} / \mathrm{kg} / \mathrm{j})\end{array}$ & $\begin{array}{r}26,8( \pm 12,8) \\
3,2( \pm 1,5) \\
1,0( \pm 0,4) \\
0,9( \pm 0,4)\end{array}$ & $\begin{array}{l}29,4( \pm 9,4) \\
3,6( \pm 1,1) \\
1,1( \pm 0,3) \\
1,0( \pm 0,3)\end{array}$ & $\begin{array}{l}- \\
- \\
-\end{array}$ \\
\hline
\end{tabular}

+ : analyse effectuée sur $n=47$ patients (données anthropométriques manquantes) 
Critère cholestase 3

L'incidence de survenue des $A B H$ selon le critère cholestase 3 était de 4,1\%. L'effectif était trop faible pour réaliser des analyses comparatives entre les cas incidents et les autres patients (Tableau 11). 
Tableau 12 - Comparaison des patients avec et sans anomalies du bilan hépatique incidentes selon le critère stéatose après introduction de la nutrition parentérale

\begin{tabular}{|c|c|c|c|}
\hline & $\begin{array}{c}\text { Oui } \\
\mathrm{n}=38(61.3 \%)\end{array}$ & $\begin{array}{c}\text { Non } \\
\mathrm{n}=\mathbf{2 4}(\mathbf{3 8 . 7 \% )} \\
\end{array}$ & $\mathbf{p}$ \\
\hline Age (années) & $60( \pm 14,2)$ & $65( \pm 15,6)$ & 0,1 \\
\hline $\begin{array}{ll}\text { Sexe } & \\
& \text { Homme } \\
& \text { Femme }\end{array}$ & $\begin{array}{l}18(47,4 \%) \\
20(52,6 \%)\end{array}$ & $\begin{array}{l}19(79,2 \%) \\
5(20,8 \%)\end{array}$ & 0,01 \\
\hline $\begin{array}{l}\text { IMC moyen }\left(\mathbf{k g} / \mathrm{m}^{2}\right) \dagger \\
\text { Dénutrition sévère selon IMC } \\
\text { Dénutrition modérée selon IMC } \\
\text { Normal } \\
\text { Surpoids } \\
\text { Obésité }\end{array}$ & $\begin{array}{c}21,5( \pm 5,5) \\
9(25,7 \%) \\
5(14,3 \%) \\
14(40,0 \%) \\
4(11,4 \%) \\
3(8,6 \%)\end{array}$ & $\begin{array}{c}21,4( \pm 5,0) \\
7(29,2 \%) \\
3(12,5 \%) \\
10(41,7 \%) \\
3(12,5 \%) \\
1(4,2 \%)\end{array}$ & $\begin{array}{l}0,9 \\
0,6 \\
0,9 \\
0,8 \\
0,8 \\
0,5\end{array}$ \\
\hline $\begin{array}{l}\text { Dénutrition selon la HAS † } \\
\text { Modérée } \\
\text { Sévère }\end{array}$ & $\begin{array}{c}3(8,6 \%) \\
10(28,6 \%)\end{array}$ & $\begin{array}{l}3(12,5 \%) \\
10(41,7 \%)\end{array}$ & $\begin{array}{l}0,5 \\
0,2\end{array}$ \\
\hline $\begin{array}{l}\text { Pathologie principale } \\
\text { Cancer } \\
\text { Pathologie hépato-bilio-pancréatique bénigne } \\
\text { Autre pathologie digestive } \\
\text { Autre }\end{array}$ & $\begin{array}{c}16(42,1 \%) \\
8(21,1 \%) \\
12(31,6 \%) \\
2(5,3 \%) \\
\end{array}$ & $\begin{array}{c}6(25 \%) \\
6(25 \%) \\
10(41,7 \%) \\
2(8,3 \%) \\
\end{array}$ & $\begin{array}{l}- \\
0,1 \\
0,8 \\
0,6\end{array}$ \\
\hline $\begin{array}{l}\text { Antécédent de chirurgie du tube digestif } \\
\text { Résection iléale } \\
\text { Résection colique } \\
\text { Résection iléo-colique } \\
\text { Chirurgie sus mésocolique }\end{array}$ & $\begin{array}{l}12(31,6 \%) \\
4(10,5 \%) \\
3(7,9 \%) \\
1(2,6 \%) \\
4(10,5 \%)\end{array}$ & $\begin{array}{c}4(16,7 \%) \\
1(4,2 \%) \\
1(4,2 \%) \\
1(4,2 \%) \\
1(4,2 \%)\end{array}$ & $\begin{array}{l}0,2 \\
0,5 \\
0,7 \\
0,4\end{array}$ \\
\hline Antécédent de cholécystectomie & $6(15,8 \%)$ & $2(8,3 \%)$ & 0,4 \\
\hline $\begin{array}{l}\text { Insuffisance intestinale } \\
\text { Grêle court } \\
\text { Fistule intestinale } \\
\text { Trouble moteur } \\
\text { Obstruction } \\
\text { Atteinte muqueuse } \\
\end{array}$ & $\begin{array}{c}17(44,7 \%) \\
6(15,8 \%) \\
3(7,9 \%) \\
5(13,2 \%) \\
7(18,4 \%) \\
0(0 \%) \\
\end{array}$ & $\begin{array}{c}14(58,3 \%) \\
2(8,3 \%) \\
0(0 \%) \\
3(12,5 \%) \\
4(16,7 \%) \\
1(4,2 \%) \\
\end{array}$ & $\begin{array}{l}- \\
0,9 \\
0,9 \\
0,9 \\
0,9 \\
0,9\end{array}$ \\
\hline NP exclusive & $13(34,2 \%)$ & $3(12,5 \%)$ & 0,06 \\
\hline Longueur moyenne de colon restant (\%) & $90 \%( \pm 10 \%)$ & $90 \%( \pm 20 \%)$ & 0,6 \\
\hline $\begin{array}{l}\text { Sepsis } \\
\qquad \text { Dont sepsis d'origine hépatobiliaire }\end{array}$ & $\begin{array}{c}16(42,1 \%) \\
3(7,9 \%)\end{array}$ & $\begin{array}{l}12(50 \%) \\
3(12,5 \%)\end{array}$ & $\begin{array}{l}0,5 \\
0,5\end{array}$ \\
\hline $\begin{array}{l}\text { Pathologie hépatobiliaire } \\
\text { Dont pathologie lithiasique }\end{array}$ & $\begin{array}{c}13(34,2 \%) \\
3(7,9 \%) \\
\end{array}$ & $\begin{array}{l}7(29,2 \%) \\
4(16,7 \%)\end{array}$ & $\begin{array}{l}0,6 \\
0,2\end{array}$ \\
\hline $\begin{array}{l}\text { Traitements hépatotoxiques } \\
\text { Antibiothérapie } \\
\text { Chimiothérapie }\end{array}$ & $\begin{array}{l}15(39,5 \%) \\
9(23,7 \%) \\
6(15,8 \%) \\
\end{array}$ & $\begin{array}{c}13(54,2 \%) \\
11(45,8 \%) \\
2(8,3 \%)\end{array}$ & $\begin{array}{c}0,6 \\
0,07\end{array}$ \\
\hline $\begin{array}{l}\text { Apports caloriques parentéraux moyens }(\mathrm{kcal} / \mathrm{kg} / \mathrm{j}) \\
\text { Dont glucides }(\mathrm{g} / \mathrm{kg} / \mathrm{j}) \\
\text { Dont protides }(\mathrm{g} / \mathrm{kg} / \mathrm{j}) \\
\text { Dont lipides }(\mathrm{g} / \mathrm{kg} / \mathrm{j})\end{array}$ & $\begin{array}{l}30,5( \pm 11,5) \\
3,7( \pm 1,4) \\
1,1( \pm 0,4) \\
1,0( \pm 0,4)\end{array}$ & $\begin{array}{l}27,4( \pm 9,9) \\
3,4( \pm 1,2) \\
1,0( \pm 0,9) \\
0,9( \pm 0,9)\end{array}$ & $\begin{array}{l}0,2 \\
0,3 \\
0,2 \\
0,2\end{array}$ \\
\hline
\end{tabular}

+ : analyse effectuée sur $n=47$ patients (données anthropométriques manquantes) 


\section{Critère stéatose}

L'incidence de survenue des $A B H$ selon le critère stéatose était de $61,3 \%$. Il existait une proportion plus importante d'homme chez les patients sans $A B H$ selon ce critère après introduction de la NP $(47,4 \%$ vs. $79,2 \%, p=0,01)$. Les patients avec $A B H$ avaient également, de manière non significative, d'avantage de patient sous NP exclusive $(34,2 \%$ vs. $12,5 \%, p=0,06)$ et d'avantage de patient sous chimiothérapie $(15,8 \%$ vs. $8,3 \%, p=0,07)$ (Tableau 12). 
Tableau 13 - Comparaison des patients avec et sans anomalies du bilan hépatique incidentes selon le critère mixte après introduction de la nutrition parentérale

\begin{tabular}{|c|c|c|c|}
\hline & $\begin{array}{c}\text { Oui } \\
n=13(36.1 \%)\end{array}$ & $\begin{array}{c}\text { Non } \\
n=24(63.9 \%)\end{array}$ & $\mathrm{p}$ \\
\hline Age (années) & $61( \pm 12,6)$ & $61( \pm 16,2)$ & 0,8 \\
\hline $\begin{array}{ll}\text { Sexe } & \\
& \text { Homme } \\
\text { Femme }\end{array}$ & $\begin{array}{l}8(61,5 \%) \\
5(38,5 \%)\end{array}$ & $\begin{array}{c}15(62,5 \%) \\
9(37,5 \%)\end{array}$ & 0,8 \\
\hline $\begin{array}{l}\text { IMC moyen }\left(\mathbf{k g} / \mathrm{m}^{2}\right)^{\dagger} \\
\text { Dénutrition sévère selon IMC } \\
\text { Dénutrition modérée selon IMC } \\
\text { Normal } \\
\text { Surpoids } \\
\text { Obésité }\end{array}$ & $\begin{array}{c}21,3( \pm 7,4) \\
4(30,8 \%) \\
1(7,7 \%) \\
5(46,2 \%) \\
1(7,7 \%) \\
1(92,3 \%)\end{array}$ & $\begin{array}{l}22,9( \pm 5,6) \\
5(21,7 \%) \\
4(17,3 \%) \\
7(30,4 \%) \\
4(17,3 \%) \\
3(13,0 \%)\end{array}$ & $\begin{array}{l}0,9 \\
0,3 \\
0,4 \\
0,6 \\
0,4 \\
0,6\end{array}$ \\
\hline $\begin{array}{l}\text { Dénutrition selon la HAS † } \\
\text { Modérée } \\
\text { Sévère }\end{array}$ & $\begin{array}{c}1(7,7 \%) \\
4(30,8 \%)\end{array}$ & $\begin{array}{l}4(17,3 \%) \\
5(21,7 \%)\end{array}$ & $\begin{array}{l}0,4 \\
0,3\end{array}$ \\
\hline $\begin{array}{l}\text { Pathologie principale } \\
\text { Cancer } \\
\text { Pathologie hépato-bilio-pancréatique bénigne } \\
\text { Autre pathologie digestive } \\
\text { Autre }\end{array}$ & $\begin{array}{c}5(38,5 \%) \\
3(23,1 \%) \\
5(38,5 \%) \\
0(0 \%)\end{array}$ & $\begin{array}{c}7(29,2 \%) \\
3(12,5 \%) \\
11(45,8 \%) \\
3(12,5 \%)\end{array}$ & $\begin{array}{l}- \\
0,9 \\
0,9 \\
0,9\end{array}$ \\
\hline $\begin{array}{l}\text { Antécédent de chirurgie du tube digestif } \\
\text { Résection iléale } \\
\text { Résection colique } \\
\text { Résection iléo-colique } \\
\text { Chirurgie sus mésocolique }\end{array}$ & $\begin{array}{c}5(38,5 \%) \\
0(0 \%) \\
3(23,1 \%) \\
1(7,7 \%) \\
1(7,7 \%) \\
\end{array}$ & $\begin{array}{c}7(29,2 \%) \\
4(16,7 \%) \\
1(4,2 \%) \\
0(0 \%) \\
2(8,3 \%) \\
\end{array}$ & $\begin{array}{l}0,9 \\
0,9 \\
0,9 \\
0,9\end{array}$ \\
\hline Antécédent de cholécystectomie & $1(7,7 \%)$ & $4(16,7 \%)$ & 0,4 \\
\hline $\begin{array}{l}\text { Insuffisance intestinale } \\
\text { Grêle court } \\
\text { Fistule intestinale } \\
\text { Trouble moteur } \\
\text { Obstruction } \\
\text { Atteinte muqueuse }\end{array}$ & $\begin{array}{c}5(38,5 \%) \\
2(15,4 \%) \\
1(7,7 \%) \\
2(15,4 \%) \\
3(23,1 \%) \\
0(0 \%)\end{array}$ & $\begin{array}{c}9(37,5 \%) \\
4(16,7 \%) \\
2(8,3 \%) \\
3(12,5 \%) \\
5(20,8 \%) \\
1(4,2 \%)\end{array}$ & $\begin{array}{l}- \\
0,9 \\
0,9 \\
0,9 \\
0,9 \\
0,9\end{array}$ \\
\hline NP exclusive & $5(38,5 \%)$ & $8(33,3 \%)$ & 0,6 \\
\hline Longueur moyenne de colon restant (\%) & $80 \%( \pm 20 \%)$ & $90 \%( \pm 20 \%)$ & 0,4 \\
\hline $\begin{array}{l}\text { Sepsis } \\
\text { Dont sepsis d'origine hépatobiliaire }\end{array}$ & $\begin{array}{c}6(46,2 \%) \\
0(0 \%)\end{array}$ & $\begin{array}{c}10(41,7 \%) \\
0(0 \%)\end{array}$ & 0,8 \\
\hline $\begin{array}{l}\text { Pathologie hépatobiliaire } \\
\text { Dont pathologie lithiasique }\end{array}$ & $\begin{array}{c}4(30,8 \%) \\
0(0 \%)\end{array}$ & $\begin{array}{c}0(0 \%) \\
1(4,2 \%)\end{array}$ & $\begin{array}{c}0,9 \\
- \\
\end{array}$ \\
\hline $\begin{array}{l}\text { Traitements hépatotoxiques } \\
\text { Antibiothérapie } \\
\text { Chimiothérapie }\end{array}$ & $\begin{array}{l}5(38,5 \%) \\
3(23,1 \%) \\
2(15,4 \%)\end{array}$ & $\begin{array}{c}8(33,3 \%) \\
7(29,2 \%) \\
1(4,2 \%)\end{array}$ & $\begin{array}{l}0,5 \\
0,3\end{array}$ \\
\hline $\begin{array}{l}\text { Apports caloriques parentéraux moyens }(\mathrm{kcal} / \mathrm{kg} / \mathrm{j}) \\
\text { Dont glucides }(\mathrm{g} / \mathrm{kg} / \mathrm{j}) \\
\text { Dont protides }(\mathrm{g} / \mathrm{kg} / \mathrm{j}) \\
\text { Dont lipides }(\mathrm{g} / \mathrm{kg} / \mathrm{j})\end{array}$ & $\begin{array}{l}33,8( \pm 18,5) \\
4,0( \pm 2,2) \\
1,2( \pm 0,7) \\
1,1( \pm 0,6)\end{array}$ & $\begin{array}{l}29,8( \pm 7,1) \\
3,7( \pm 0,7) \\
1,1( \pm 0,2) \\
1,0( \pm 0,2)\end{array}$ & $\begin{array}{l}0,4 \\
0,4 \\
0,4 \\
0,4\end{array}$ \\
\hline
\end{tabular}

$\dagger$ : analyse effectuée sur $n=47$ patients (données anthropométriques manquantes) 
Critère mixte

L'incidence de survenue des $A B H$ selon le critère cholestase 1 était de 36,1\%. II n'y avait pas de différence significative entre les patients avec $A B H$ et sans $A B H$ après introduction de la NP (Tableau 13). 


\section{Discussion}

Notre étude met en évidence, qu'à l'exception du critère stéatose, 30 à $50 \%$ des malades requérant une NP présentaient des $\mathrm{ABH}$ avant même le début de celle-ci.

La cause principale (pour la quasi-totalité des critères) est l'existence d'une pathologie hépatobilio-pancréatique (angiocholite, cholécystite, pancréatite, hépatite alcoolique, virale...). La structure de recrutement (services d'hépato-gastroentérologie) explique sans doute cette forte prévalence.

La prévalence médiane des $A B H$ après introduction de la NP était de $42,7 \%$ (IQ = 39,0 - 56,1 $\%)$. La prévalence des $A B H$ ne s'aggravait pas de manière significative après introduction de la NP, excepté selon le critère stéatose $(10,1 \%$ vs. $59,8 \%, p<0,01)$. Ce résultat rejoint celui de Sasdelli et al. qui montrait que la majorité des patients sous NP au long cours ne perturbait pas ou peu son bilan hépatique (8). Concernant le critère stéatose, qui a un seuil extrêmement bas (ALAT et ASAT au-delà de la limite supérieure de la normale), Sorbi et al. ont étudié le rapport ASAT/ALAT comme outil pour différentier la NASH (Non Alcoholic Steato-Hepatitis), avec un rapport inférieur à 1, de la maladie chronique du foie liée à l'alcool, avec un rapport supérieur à 1, avec une bonne sensibilité et une bonne spécificité après exclusion des autres causes d'hépatopathie (22). Le fait que la prévalence évolue de manière significative conduit à confirmer que diverses anomalies anatomiques hépatobiliaires, distinctes de celles liées à l'alcool, et possiblement en rapport avec les apports nutritionnels glucidiques et lipidiques, se produisent sous NP ; on peut s'interroger sur la pertinence clinique de ce critère extrêmement sensible.

L'incidence des $\mathrm{ABH}$ dans notre étude variait de $4,1 \%$ à $61,3 \% \%$ selon le critère biologique choisi, avec une incidence médiane à $28,9 \%(I Q=23,4-34,0 \%)$. Cette donnée est cohérente avec la littérature s'intéressant à l'incidence de l'IFALD puisque que cette dernière varie entre 7 et $65 \%$ selon les études et les critères diagnostiques (8-10).

Les cas incidents avaient un IMC significativement plus bas pour le critère cholestase 1 (18,3 $\pm 4,5 \mathrm{~kg} / \mathrm{m}^{2}$ vs. $23,2 \pm 6,1 \mathrm{~kg} / \mathrm{m}^{2}$ ) et avaient également une proportion plus importante de patients avec un IMC répondant à la définition HAS de la dénutrition sévère de manière significative pour le critère cholestase 1 ( $58,3 \%$ vs. $20,0 \%$ ) et non significative pour le critère cholestase 2 (50,0 \% vs. $16,1 \%)$. Ils avaient également significativement plus d'insuffisance intestinale pour le critère cytolyse $(66.7 \%$ vs. $35.3 \%, p=0,04)$.

Le lien entre dénutrition et IFALD est bien décrit dans la littérature. Un des mécanismes physiopathologiques possibles serait l'existence d'un déficit en choline induit par une carence d'apport et/ou un défaut de synthèse de cette dernière car elle est issue d'un premier passage hépatique de la méthionine $(7,11)$. Des apports oraux et entéraux insuffisants pourraient perturber le microbiote et ainsi via une hyperperméabilité intestinale favoriser l'endotoxémie et les lésions hépatiques $(16,17)$. Le jeûne altère également la synthèse, la qualité et la circulation des acides biliaires et favorise ainsi la cholestase (12). Les perturbations du bilan hépatique peuvent se voir chez les patients dénutris sévères, notamment les anorexiques mentales, lorsque l'autophagie hépatique apparait $(23,24)$. Enfin, et de manière certainement plus pertinente, le syndrome de renutrition, observé lors du début d'une nutrition artificielle chez des malades chroniquement et sévèrement dénutris, peut s'accompagner d'ABH (25).

D'autres facteurs tendaient vers une association positive sans significativité statistique et allaient également dans le sens de la littérature sur I'IFALD : la durée de la NP associée aux ABH incidentes selon le critère cholestase $1(23,6 \pm 12,2$ j vs. $16,5 \pm 10,8, p=0,08)$ et la NP exclusive associée aux $\mathrm{ABH}$ incidentes selon le critère stéatose $(34,2 \%$ vs. $12,5 \%, p=0,06)$. 
La proportion plus importante de femmes chez les patients avec $A B H$ incidentes selon le critère stéatose $(52,6 \%$ vs. $20,8 \%, p=0,01)$ est d'interprétation plus difficile. II n'existe pas d'élément dans la littérature suggérant une susceptibilité à la stéatose dépendante du sexe.

Ces résultats favorables sont sans doute liés à la qualité de la prescription de NP dans des services où la majorité des médecins et diététiciennes a été formée à la nutrition médicale. Outre des apports moyens raisonnables de $30 \mathrm{kcal} / \mathrm{kg} / \mathrm{j}$, la nutrition parentérale utilisée était dans l'écrasante majorité de l'Olimel N7E ${ }^{\circledR}$ (Baxter, Guyancourt, France) dont les lipides sont issus à $80 \%$ d'huile d'olive source d'oméga-9 et à $20 \%$ d'huile de soja source d'oméga-9 et oméga-6. L'utilisation quasi exclusive de cette émulsion lipidique, moins riche en oméga- 6 que les émulsions lipidiques d'anciennes générations peut expliquer l'absence d'imputabilité significative de la NP aux ABH dans notre étude. De fait, aucun patient ne recevait d'INTRALIPIDE $20 \%{ }^{\circledR}$ (Fresenius-Kabi, Sèvres, France), émulsion lipidique à base d'huile de soja purifiée (acides gras essentiels). La littérature suggère de favoriser les émulsions lipidiques à base d'huile de poisson car leurs meilleur rapport oméga-6/oméga-3 limite l'atteinte hépatique. Ceci pourrait être dû à un effet sur les médiateurs de l'inflammation $(19,26,27)$. Les apports lipidiques pour éviter la survenue d'une IFALD doivent être inférieur à $1 \mathrm{~g} / \mathrm{kg} / \mathrm{jour}$; dans notre étude, les apports moyens étaient évalués à $1,0 \pm 0,3 \mathrm{~g} / \mathrm{kg} / \mathrm{jour}$, conformes aux recommandations.

Notre étude est la première, à notre connaissance, à s'intéresser aux $\mathrm{ABH}$ précoces chez les patients sous NP d'introduction récente. L'étude était exhaustive puisque tous les malades respectant les critères d'inclusion et de non-inclusion ont été inclus durant la période. L'indication de la NP était variée et non limitée à l'insuffisance intestinale, puisque cette dernière ne concernait que $53,7 \%$ des patients. Il s'agit là d'un des points forts de notre étude puisqu'elle a inclus des patients qui n'avaient pas nécessairement une indication formelle à une NP en première intention, ce qui est malheureusement assez fidèle à la pratique clinique souvent observée. De même, $60 \%$ des malades n'étaient pas dénutris, bien que leurs pathologies les rendaient à risque de dénutrition. Un autre point fort de notre étude est que nous avons comparé les bilans biologiques avant et après introduction de la NP pour ne compter comme cas incidents que les patients dont les ABH étaient d'apparition nouvelle mais probablement au prix d'une difficulté à mettre en évidence des différences significatives. De plus, le recueil du bilan biologique avant introduction de la NP, même s'il n'était pas toujours disponible, a permis d'évaluer l'incidence et d'estimer l'imputabilité de la NP récemment introduite aux ABH.

Notre étude présentait plusieurs limites. D'abord, l'effectif était limité (82 patients) et pour 12 à 13 d'entre eux nous n'avions pas à disposition de bilan biologique datant d'avant l'introduction de la $\mathrm{NP}$, ne permettant pas de qualifier leurs $\mathrm{ABH}$ d'incidentes. La présence de près de la moitié de patients sous NP sans insuffisance intestinale (nutrition entérale fréquemment non proposée ou refusée par le patient, rares complications de la nutrition entérale), même si cela correspond à une réalité, est probablement une source de biais puisque que la littérature suggère que les anomalies hépatiques sont, chez les patients sous NP au long cours, directement ou indirectement corrélées à l'insuffisance intestinale. De ce fait, on ne peut probablement pas sur l'effectif de patients avec insuffisance intestinale qualifier d'IFALD les ABH sous NP.

Une autre limite de notre étude était l'absence de recueil des ingesta qui ne permettait pas donc d'identifier formellement les patients « surnutris ». Or les apports glucidiques et lipidiques trop importants par rapport aux besoins sont reliés à la survenue de I'IFALD dans la littérature.

Nous n'avions enfin pas de donnée morphologique concomitante aux $A B H$ (échographie, scanner ou IRM) pour la majorité des malades, et aucune donnée histologique. Pour autant, l'apport d'une preuve anatomopathologique d'IFALD développée sous NP au long cours n'étant pas 
recommandée, il parait peu licite et éthique de réaliser une biopsie hépatique en cas de survenue d'ABH mineures et non graves d'apparition aigues.

En conclusion, la NP moderne, administrée de manière cyclique, quantitativement et qualitativement adaptée au patient ne parait pas être significativement associée aux à la survenue d'ABH précoces. La survenue d'ABH en début de NP doit faire rechercher d'autres causes que la NP, en particulier une pathologies hépato-bilio-pancréatique. II est par ailleurs probable que ces $A B H$, lorsqu'elles sont en rapport avec la NP, traduisent un syndrome de renutrition et n'aient pas de rapport avec l'insuffisance intestinale; on pourrait alors, en aigu, parler de PNALD (Parenteral Nutrition Associated Liver Disease) et pas d'IFALD. II serait intéressant de suivre ces malades plus longtemps, y compris après l'arrêt de la NP lorsqu'elle est de courte à moyenne durée, afin d'évaluer les conséquences de ces $\mathrm{ABH}$. De ce fait, tout comme en situation chronique, il parait utile d'établir un consensus sur les paramètres biologiques traduisant une atteinte anatomique pertinente. 


\section{Bibliographie}

1. Pironi L, Arends J, Bozzetti F, Cuerda C, Gillanders L, Jeppesen PB, et al. ESPEN guidelines on chronic intestinal failure in adults. Clin Nutr. avr 2016;35(2):247-307.

2. Joly F, Baxter J, Staun M, Kelly DG, Hwa YL, Corcos O, et al. Five-year survival and causes of death in patients on home parenteral nutrition for severe chronic and benign intestinal failure. Clin Nutr. août 2018;37(4):1415-22.

3. Pironi L, Arends J, Baxter J, Bozzetti F, Peláez RB, Cuerda C, et al. ESPEN endorsed recommendations. Definition and classification of intestinal failure in adults. Clin Nutr. avr 2015;34(2):171-80.

4. Bielawska B, Allard J. Parenteral Nutrition and Intestinal Failure. Nutrients. 6 mai 2017;9(5):466.

5. Pironi L, Konrad D, Brandt C, Joly F, Wanten G, Agostini F, et al. Clinical classification of adult patients with chronic intestinal failure due to benign disease: An international multicenter crosssectional survey. Clin Nutr. avr 2018;37(2):728-38.

6. Lal S, Pironi L, Wanten G, Arends J, Bozzetti F, Cuerda C, et al. Clinical approach to the management of Intestinal Failure Associated Liver Disease (IFALD) in adults: A position paper from the Home Artificial Nutrition and Chronic Intestinal Failure Special Interest Group of ESPEN. Clin Nutr. déc 2018;37(6):1794-7.

7. Buchman A, Naini B, Spilker B. The Differentiation of Intestinal-Failure-Associated Liver Disease from Nonalcoholic Fatty Liver and Nonalcoholic Steatohepatitis. Semin Liver Dis. 15 févr 2017;37(01):033-44.

8. Sasdelli AS, Agostini F, Pazzeschi C, Guidetti M, Lal S, Pironi L. Assessment of Intestinal Failure Associated Liver Disease according to different diagnostic criteria. Clin Nutr. juin 2019;38(3):1198-205.

9. Cavicchi M, Beau P, Crenn P, Degott C, Messing B. Prevalence of Liver Disease and Contributing Factors in Patients Receiving Home Parenteral Nutrition for Permanent Intestinal Failure. Ann Intern Med. 4 avr 2000;132(7):525.

10. Rochling FA, Catron HA. Intestinal failure-associated liver disease: causes, manifestations and therapies. Curr Opin Gastroenterol. mars 2019;35(2):126-33.

11. Mitra A, Ahn J. Liver Disease in Patients on Total Parenteral Nutrition. Clin Liver Dis. nov 2017;21(4):687-95.

12. Bond A, Huijbers A, Pironi L, Schneider SM, Wanten G, Lal S. Review article: diagnosis and management of intestinal failure-associated liver disease in adults. Aliment Pharmacol Ther. sept 2019;50(6):640-53.

13. Luman W, Shaffer JL. Prevalence, outcome and associated factors of deranged liver function tests in patients on home parenteral nutrition. Clin Nutr Edinb Scotl. août 2002;21(4):337-43.

14. Van Gossum A, Pironi L, Messing B, Moreno C, Colecchia A, D’Errico A, et al. Transient Elastography (FibroScan) Is Not Correlated With Liver Fibrosis but With Cholestasis in Patients With Long-Term Home Parenteral Nutrition. J Parenter Enter Nutr. août 2015;39(6):719-24. 
15. Beath SV, Kelly DA. Total Parenteral Nutrition-Induced Cholestasis. Clin Liver Dis. févr 2016;20(1):159-76.

16. Cahova M, Bratova M, Wohl P. Parenteral Nutrition-Associated Liver Disease: The Role of the Gut Microbiota. Nutrients. 7 sept 2017;9(9):987.

17. Hvas C, Kodjabashia K, Nixon E, Hayes S, Farrer K, Abraham A, et al. Reversal of intestinal failureassociated liver disease (IFALD): emphasis on its multifactorial nature. Frontline Gastroenterol. avr 2016;7(2):114-7.

18. Zugasti Murillo A, Petrina Jáuregui E, Elizondo Armendáriz J. Hepatopatía asociada a nutrición parenteral y emulsiones lipídicas. Endocrinol Nutr. juin 2015;62(6):285-9.

19. Nandivada P, Carlson SJ, Chang MI, Cowan E, Gura KM, Puder M. Treatment of Parenteral Nutrition-Associated Liver Disease: The Role of Lipid Emulsions. Adv Nutr. 1 nov 2013;4(6):711-7.

20. Cummings JH, James WP, Wiggins HS. Role of the colon in ileal-resection diarrhoea. Lancet Lond Engl. 17 févr 1973;1(7799):344-7.

21. Diagnostic de la dénutrition de l'enfant et de l'adulte - Fiche outil - Novembre 2019 [Internet]. Disponible sur: https://www.has-sante.fr/upload/docs/application/pdf/201911/fiche_outil_diagnostic_denutrition.pdf

22. Sorbi D. The Ratio of Aspartate Aminotransferase to Alanine Aminotransferase: Potential Value in Differentiating Nonalcoholic Steatohepatitis From Alcoholic Liver Disease. 1999;94(4):5.

23. Kheloufi M, Boulanger CM, Durand F, Rautou P-E. Liver Autophagy in Anorexia Nervosa and Acute Liver Injury. BioMed Res Int. 2014;2014:1-10.

24. Rosen E, Bakshi N, Watters A, Rosen HR, Mehler PS. Hepatic Complications of Anorexia Nervosa. Dig Dis Sci. nov 2017;62(11):2977-81.

25. Nishioka H, Yoshizaki A, Imai Y, Higashibeppu N. Starvation-induced Liver Enzyme Elevation after Initiation of Feeding. Intern Med. 1 mars 2019;58(5):749-53.

26. Fu R-H. Selection of lipid emulsions for protection against parenteral nutrition-associated liver disease. Pediatr Neonatol. août 2019;60(4):355-6.

27. Tillman EM. Review and Clinical Update on Parenteral Nutrition-Associated Liver Disease. Nutr Clin Pract. févr 2013;28(1):30-9. 


\section{Serment d'Hippocrate}

“Au moment d'être admis à exercer la médecine, je promets et je jure d'être fidèle aux lois de I’honneur et de la probité.

Mon premier souci sera de rétablir, de préserver ou de promouvoir la santé dans tous ses éléments, physiques et mentaux, individuels et sociaux.

Je respecterai toutes les personnes, leur autonomie et leur volonté, sans aucune discrimination selon leur état ou leurs convictions. J'interviendrai pour les protéger si elles sont affaiblies, vulnérables ou menacées dans leur intégrité ou leur dignité. Même sous la contrainte, je ne ferai pas usage de mes connaissances contre les lois de l'humanité.

J'informerai les patients des décisions envisagées, de leurs raisons et de leurs conséquences.

Je ne tromperai jamais leur confiance et n'exploiterai pas le pouvoir hérité des circonstances pour forcer les consciences.

Je donnerai mes soins à l'indigent et à quiconque me les demandera. Je ne me laisserai pas influencer par la soif du gain ou la recherche de la gloire.

Admis(e) dans l'intimité des personnes, je tairai les secrets qui me seront confiés. Reçu(e) à I'intérieur des maisons, je respecterai les secrets des foyers et ma conduite ne servira pas à corrompre les mœurs.

Je ferai tout pour soulager les souffrances. Je ne prolongerai pas abusivement les agonies. Je ne provoquerai jamais la mort délibérément.

Je préserverai l'indépendance nécessaire à l'accomplissement de ma mission. Je n'entreprendrai rien qui dépasse mes compétences. Je les entretiendrai et les perfectionnerai pour assurer au mieux les services qui me seront demandés.

J'apporterai mon aide à mes confrères ainsi qu'à leurs familles dans l'adversité.

Que les hommes et mes confrères m'accordent leur estime si je suis fidèle à mes promesses ; que je sois déshonoré(e) et méprisé(e) si j’y manque." 



\section{Résumé}

Introduction: L'IFALD (Intestinal Failure Associated Liver Disease) désigne les atteintes hépatiques multifactorielles observées chez des patients souffrant d'insuffisance intestinale sous nutrition parentérale (NP). Si I'IFALD est mieux connue, Il existe peu de littérature sur les anomalies du bilan hépatique $(A B H)$ chez les patients sous NP d'introduction récente.

Patients et méthodes: L'objectif de notre étude était de déterminer la prévalence et l'incidence des $\mathrm{ABH}$ chez des patients sous NP, ainsi que les facteurs associés à la survenue de ces anomalies. Tous les patients hospitalisés dans les services d'Hépato-gastroentérologie et Nutrition du Centre Hospitalier Universitaire de Nice sous NP depuis plus 7 jours et moins de 3 mois ont été inclus de manière consécutive de mai 2019 à mai 2020. Nous avons collecté les données anthropométriques, l'état nutritionnel, les antécédents et pathologies évolutives des patients, la prise de traitements hépatotoxiques et les modalités d'administration de la nutrition parentérale. Le bilan biologique du jour de l'inclusion était recueilli ainsi que, si disponible, le dernier bilan réalisé la semaine précédant I'introduction de la NP. Nous avons sélectionné et comparé six critères biologiques déjà utilisées dans la littérature pour I'IFALD.

Résultats : 82 malades ont été inclus et l'incidence des $A B H$ a pu être évaluée chez 70 d'entre eux. La prévalence des $A B H$ avant introduction de la NP variait de $10,7 \%$ à $47,1 \%$ et était, pour presque chaque critère, significativement associée à des pathologies hépato-bilio-pancréatiques. La prévalence médiane des ABH après introduction de la NP était de 42,7 \% (IQ = 39,0 - 56,1\%). La prévalence n'augmentait pas de manière significative après introduction de la NP excepté selon le critère stéatose $(10,1 \%$ vs. $59,8 \%, p<0,01)$. L'incidence des $A B H$ dans notre étude variait de $4,1 \%$ à $61,3 \%$ selon le critère biologique choisi, avec une incidence médiane à $28,9 \%(\mathrm{IQ}=23,4-34,0 \%)$. Les cas incidents selon I'un des critères de cholestase avaient un IMC significativement plus bas $18,3 \pm 4,5$ vs. $23,2 \pm 6,1$ $\mathrm{kg} / \mathrm{m}^{2}$ ) et répondaient à la définition HAS de la dénutrition sévère ( $\left.58,3 \% \mathrm{vs} .20,0 \%\right)$. Les cas incidents selon le critère de cytolyse avaient davantage d'insuffisance intestinale $(66.7 \%$ vs. $35.3 \%, p=0,04)$.

Conclusion : La NP moderne ne parait pas être significativement associée à la survenue d'ABH précoces. La découverte d'ABH en début de NP doit orienter en premier lieu vers une pathologie hépato-bilio-pancréatique, mais doit aussi faire évoquer un syndrome de renutrition chez un malade (sévèrement) dénutri. 
